(C) The Author(s), 2021. Published by Cambridge University Press on behalf of The Nutrition Society. This is an Open Access article, distributed under the terms of the Creative Commons Attribution licence (https://creativecommons.org/licenses/by/4.0/), which permits unrestricted re-use, distribution, and reproduction in any medium, provided the original work is properly cited.

\title{
Functional properties of edible insects: a systematic review
}

\author{
V. D’Antonio, N. Battista, G. Sacchetti, C. Di Mattia and M. Serafini* (1) \\ Faculty of Bioscience and Technology for Agriculture, Food and Environment, University of Teramo, Via Balzarini 1, 64100 \\ Teramo, Italy
}

\section{Abstract}

Consumption of edible insects has been widely suggested as an environmentally sustainable substitute for meat to reduce greenhouse gas emissions. However, the novel research field for edible insects relies on the content of bioactive ingredients and on the ability to induce a functional effect in humans. The goal of this manuscript is to review the available body of evidence on the properties of edible insects in modulating oxidative and inflammatory stress, platelet aggregation, lipid and glucose metabolism and weight control. A search for literature investigating the functional role of edible insects was carried out in the PubMed database using specific keywords. A total of 55 studies, meeting inclusion criteria after screening, were divided on the basis of the experimental approach: in vitro studies, cellular models/ex vivo studies or in vivo studies. In the majority of the studies, insects demonstrated the ability to reduce oxidative stress, modulate antioxidant status, restore the impaired activity of antioxidant enzymes and reduce markers of oxidative damage. Edible insects displayed anti-inflammatory activity reducing cytokines and modulating specific transcription factors. Results from animal studies suggest that edible insects can modulate lipid and glucose metabolism. The limited number of studies focused on the assessment of anti-coagulation activity of edible insects makes it difficult to draw conclusions. More evidence from dietary intervention studies in humans is needed to support the promising evidence from in vitro and animal models about the functional role of edible insect consumption.

\section{Key words: Antioxidants: Edible insects: Functional foods: Health: Inflammation}

(Received 23 March 2021; revised 11 October 2021; accepted 22 November 2021)

\section{Introduction}

The increasing global food demand, strictly linked to the growing world population, expected to reach almost 10 billion in
2050, has brought a new global interest on the human consumption of edible insects ${ }^{(1)}$. The consumption of insects by humans is not a novel phenomenon, having been practiced since early in human evolution, and is nowadays part of the usual diet in many

Abbreviations: 8-OHdG, 8-hydroxy-2'-deoxyguanosine; ABCA1, ATP-binding cassette transporter A1; ABCG1, ATP-binding cassette transporter G1; ABTS, 2,2'azino-bis(3-ethylbenzothiazoline-6-sulfonic acid; ACAT2, acetyl-CoA acetyltransferase 2; ACE, angiotensin-converting enzyme; ADP, adenosine diphosphate; ADRP, adipose differentiation-related protein; AGPAT1, 1-acylglycerol-3-phosphate $O$-acyltransferase 1 ; AMPK- $\alpha$, adenosine monophosphate-activated protein kinase- $\alpha$; aPTT, activated partial thromboplastin time; AUC, area under the curve; C/EBP $\alpha$, CCAAT/enhancer-binding protein $\alpha$; CA, chromosome aberration; BW, body weight; BWG, body weight gain; CAT, catalase; CD36, cluster of differentiation 36; ChREBP, carbohydrate-response element-binding protein; CLP, caecal ligation and puncture; CP, compound; CYP7A1, cholesterol $7 \alpha$ hydroxylase; DGAT1, diacylglycerol O-acyltransferase 1 ; D-HMVECs, diabetic type 2 microvascular endothelial cells; DPPH, 1,1-diphenyl-2-picrylhydrazyl; DPP-IV, dipeptidyl peptidase-4; ELOVL2, fatty acid elongase 2; ELOVL5, fatty acid elongase 5; ERK, extracellular signal-regulated kinases; ET-1, endothelin-1; F4/80+ KCs, F4/80-positive Kupffer cells; FABP1, fatty acid-binding protein 1; FADS2, fatty acid desaturase 2; FAS, fatty acid synthase; FATP5, fatty acid transport protein 5; FRAP, ferric reducing antioxidant power; FXa, factor Xa; GHG, greenhouse gas; GI, glucose index; GM-CSF, granulocyte-macrophage colony-stimulating factor; GPAT1, glycerol-3-phosphate acyltransferase 1; GPAT4, glycerol-3- phosphate acyltransferase 4; GPx, glutathione peroxidase; GR, glutathione reductase; GST, glutathione $S$-transferase; HbA1c, glycosylated haemoglobin concentration; HDL, high-density lipoprotein; HMGCR, 3-hydroxy-3-methylglutaryl-CoA reductase; HOMA-IR, homoeostasis model assessment of insulin resistance; HUVECs, human umbilical vein endothelial cells; ICAM-1, intercellular adhesion molecule-1; IFN $\alpha$, interferon $\alpha$; IFN- $\gamma$, interferon $\gamma$; IgA, immunoglobulin A; IgE, immunoglobulin E; IgG, immunoglobulin G; IgM, immunoglobulin M; IL-10, interleukin-10; IL-12, interleukin-12; IL-13, interleukin-13; IL-1 $\alpha$, interleukin-1 $\alpha$; IL-1 $\beta$, interleukin-1 $\beta$; IL-2, interleukin-2; IL-4, interleukin-4; IL-5, interleukin-5; IL-6, interleukin-6; IL-7, interleukin-7; IL-8, interleukin-8; ISI, insulin sensitivity index; ITT, insulin tolerance test; JNK, c-Jun $N$ terminal kinase; LDL, low-density lipoprotein; LPL, lipoprotein lipase; LPS, lipopolysaccharide; LS, lipo-soluble extract; LXR $\alpha$, liver X receptor; MARCKS, myristoylated alanine-rich C-kinase substrate; MDA, malondialdehyde; MN, micronucleus; MUFA, monounsaturated fatty acids; NEFA, non-esterified fatty acid; NF-кB, nuclear factor-кB; NO, nitric oxide; Nrf2, nuclear factor erythroid 2-related factor; OGTT, oral glucose tolerance test; ORAC, oxygen radical absorbance capacity; PAI-1, plasminogen activator inhibitor-1; PAP1, phosphatidate phosphatase 1 ; PH, protein hydrolysates; p-IkB- $\alpha$, phosphorylated-inhibitor of nuclear factor-kappa B-alpha; PPAR $\gamma$, peroxisome proliferator-activated receptor $\gamma$; PT, prothrombin time; PUFA, polyunsaturated fatty acids; RNS, reactive nitrogen species; ROS, reactive oxygen species; SAHR, scavenging activity on hydroxyl radicals; SCD1, stearoyl-CoA desaturase-1; SCE, sister chromatid exchange; SCFA, short-chain fatty acids; SFA, saturated fatty acids; SOD, superoxide dismutase; SREBP-1c, sterol regulatory element-binding protein 1c; SREBP-2, sterol regulatory element- binding protein 2; SRSC, superoxide radical scavenging capacity; TAC, total antioxidant capacity; TBA, total bile acid; TC, total cholesterol; TEAC, Trolox equivalent antioxidant capacity; TG, triacylglycerol; TLR4, toll-like receptor 4 ; TNF- $\alpha$, tumour necrosis factor- $\alpha$; TOS, total oxidant status; tPA, tissue plasminogen activator; TT, thrombin time; VCAM-1, vascular cell adhesion molecule-1; VEGF, vascular endothelial growth factor; WS, water-soluble extract.

* Corresponding author: Mauro Serafini, email: mserafini@unite.it 
countries ${ }^{(2)}$. Edible insects have positive environmental and economic implications and are characterised by high feed-conversion efficiency and low greenhouse gas (GHG) emissions, land use and environmental contamination ${ }^{(1)}$. Recently, consumption of edible insects has been widely suggested as an environmentally sustainable substitute for meat and animal products to reduce their extremely high global intake, responsible for massive GHG emission ${ }^{(1)}$

From a nutritional point of view, edible insects are a good source of nutrients characterised by high levels of essential amino acids, fibre, vitamin B12, iron, zinc, omega-3 and omega- 6 fatty acids, and polysaccharides and might contribute to a balanced gut microbiota ${ }^{(3)}$. However, the innovative aspect of edible insects is related to their content of bioactive ingredients and their role as functional foods, defined as foods providing a health benefit beyond basic nutrition.

Throughout human history, entomotherapy, the medicinal use of edible insects, has been used in traditional medicine in wound healing and as curative therapy for respiratory disorders and stomachache ${ }^{(4)}$. The available body of evidence on the functional role of edible insects is mainly focused on their role as antioxidant ingredients in vitro or in cellular models, as recently reviewed by D'Antonio et al. ${ }^{(5)}$. Moreover, there is scattered evidence on the role of edible insects in platelet aggregation, as antiinflammatory agents ${ }^{(6)}$ or as modulators of lipid ${ }^{(7)}$ and glucose metabolism ${ }^{(8)}$. Furthermore, the available studies tested a wide range of different insects, extracts or protein fractions, making it difficult to draw clear conclusions about their functional properties without a review of the available evidence. Recently, findings related to the nutritional, functional and health properties as well as consumer acceptance of edible insects have been thoroughly reviewed ${ }^{(9-13)}$. However, none of the manuscripts provided a comprehensive and systematic review of the available evidence on the functional roles of edible insects.

The goal of this work is to review the available body of evidence on the properties of edible insects in modulating oxidative and inflammatory stress, platelet aggregation, weight control, and lipid and glucose metabolism.

\section{Material and methods}

The search strategy of the study is shown in Figure 1. We first systematically searched PubMed database (National Library of Medicine, Bethesda, MD; https://pubmed.ncbi.nlm.nih.gov) using the following keywords: (edible insect) AND (functional) OR cardiovascular) OR platelet aggregation) OR oxidative) OR antioxidant) OR glycemia) OR diabetes) OR inflammation) OR immune) OR cholesterol) OR hypolipidemic) OR hypocholesterolemic) OR thrombosis) OR hepatoprotective) OR liver) OR adipose tissue) OR body composition) OR hypoglycaemic). The search, carried out in November 2020, with no limit ranges for the year of publication and with the 'English' filter activated, yielded 620 results (Figure 1). Reviews, systematic reviews and meta-analyses $(n=78)$ were excluded. Articles were screened to exclude those not relevant to the topics such as allergenic properties, nutrient composition, technological functionality, safety assessment, rearing, non-edible insects or other functional effects $(n=492)$. The references of the selected papers were screened, and two additional papers were identified. Furthermore, to include papers without edible insect as key word, we performed a search for the following insect names: Bombyx mori, Tenebrio molitor, cricket and grasshoppers, identifying three more papers. Fifty-five papers, focusing on oxidative and inflammatory stress, platelet aggregation, weight control, lipid and glucose metabolism, were identified.

Results are presented in Tables 1-6: in vitro studies (Tables 13), cellular models/ex vivo studies (Tables 4 and 5) and in vivo (Table 6) studies. They were further grouped by topic: antioxidant activity in vitro (Table 1 ), in vitro effects on platelet aggregation (Table 2), other in vitro effects (Table 3), antioxidant activity in cellular models or ex vivo (Table 4), and other effects in cellular models/ex vivo (Table 5). For the in vivo studies, results were divided into six categories: body and organ weight and composition, inflammatory status, oxidative/antioxidant status, lipid status, glycaemia/insulin status and coagulation markers.

\section{Results}

\section{Antioxidant activity in vitro}

Table 1 summarises the results of twenty-two studies ${ }^{(6,14-34)}$ on the in vitro antioxidant activity of different extracts from edible insects. The studies involved thirty-one species of insects, among which the most studied was Tenebrio molitor, tested in ten papers $^{(14,15,20-23,25,26,28,30)}$. Acheta domesticus and Gryllodes sigillatus were cited respectively in four and ${ }^{(14,25,26,30)}$ three papers $^{(6,15,34)}$, while Alphtobius diaperinus ${ }^{(26,29)}$, Bombyx mori $^{(26,31)}$,Hermetia illucens $^{(16,17)}$ and Lethocerus indicus ${ }^{(26,27)}$ were investigated in two papers. Water-soluble fractions were investigated in fourteen research articles ${ }^{(6,19-22,24-32)}$, while protein hydrolysates were reported in six papers ${ }^{(14-17,23,34)}$ and liposoluble fractions were tested in two papers only ${ }^{(26,33)}$. Among the several methods taken into account, the most used was the 1,1-diphenyl-2-picrylhydrazyl (DPPH) method, cited in sixteen studies $^{(6,14,15,18,20-22,24,25,27,28,30,32-35)}$, while 2,2'-azo-bis(3-ethylbenzothiazoline-6-sulfonic acid (ABTS) and ferric reducing antioxidant power (FRAP) methods were performed in thirteen $^{(6,15,16,19-21,23,25,26,28,29,34,35)}$ and nine papers $^{(17,21,22,25,26,28,31,32,34)}$, respectively. Seven studies investigated the scavenging activity of selected fractions against different radicals ${ }^{(16,17,22,24,28,29,32)}$, three involving metal ion chelating $\operatorname{activity}^{(15,25,34)}$ and one utilising $\beta$-carotene and linolenic acid bleaching tests ${ }^{(36)}$. All tested fractions showed significant antioxidant activity, the only exception being the protein hydrolysate from Gryllodes sigillatus in FRAP assay ${ }^{(34)}$. Only the study conducted by Di Mattia et al. ${ }^{(26)}$ provided a comparison between antioxidant activity of water and lipo-soluble fractions of edible insect and food extracts. More specifically, when water-soluble extracts of edible insects were analysed, Calliptamus italicus, Bombyx mori and Acheta domesticus crickets showed an antioxidant capacity, expressed as Trolox equivalent antioxidant capacity (TEAC), five-fold higher than fresh orange juice. Furthermore, water-soluble extracts of Calliptamus italicus, Imbrasia oyemensis and Acheta domesticus displayed a 


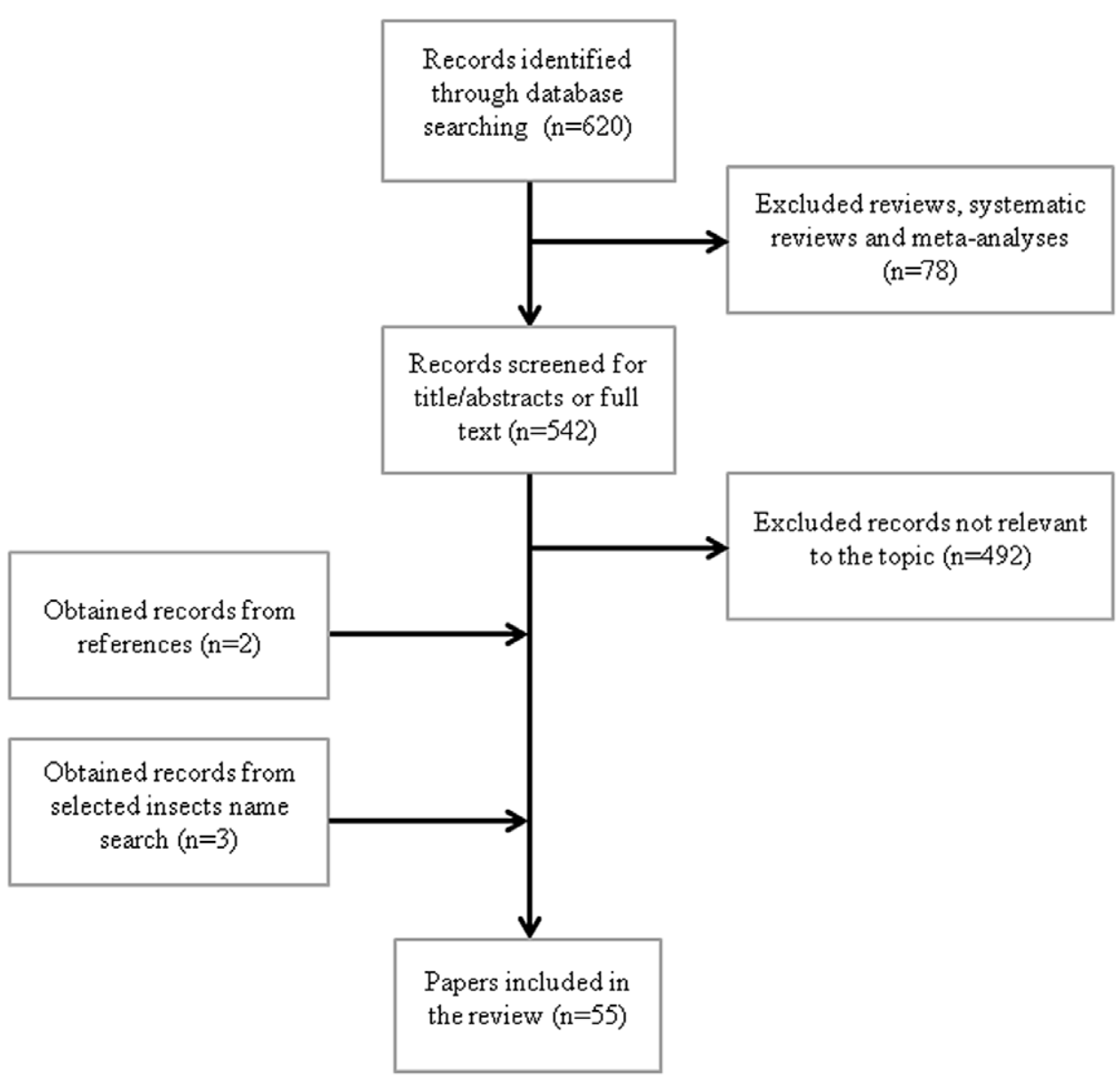

Fig. 1. Flow diagram for search strategy.

reducing power (FRAP) double that of fresh orange juice. As regards the liposoluble fraction, Bombyx mori, Tanna japonensis and Imbrasia oyemensis showed a TEAC twice that of olive oil. Regarding endogenous antioxidants, Dutta et al. (2016) showed that an aqueous extract of Vespa affinis was able to increase the activity of catalase (CAT) and glutathione $S$-transferase $(\mathrm{GST})^{(24)}$.

\section{Anti-platelet aggregation activity in vitro}

Seven edible insects were tested for their effects on coagulation markers as described in Table 2. In the study by Pyo and colleagues $(2020)^{(28)}$, ethanol extract of the edible insect Teleogryllus emma was able to prolong thrombin time (TT) and activated partial thromboplastin time (aPTT), but not prothrombin time (PT); also relative PT, TT and aPTT were increased. In the same study, Allomyrina dichotoma, Apis mellifera, Gryllus bimaculatus, Protaetia brevitarsis and Tenebrio molitor did not exert the same effect. However, all above-mentioned insects, with the exception of Apis mellifera, were able to increase platelet aggregation; conversely, haemolytic activity was observed only in Apis mellifera and Teleogryllus emma. $\mathrm{N}$-acetyl dopamine dimers obtained from Oxya chinensis sinuosa showed the ability of inhibit factor $\mathrm{Xa}$ (FXa) and to reduce platelet aggregation induced by adenosine diphosphate (ADP) or U46619, but not by thrombin. However, they did not inhibit thrombin, trypsin, elastase, plasmin, streptokinase, tissue plasminogen activator (tPA) or urokinase ${ }^{(37)}$. Indole alkaloids isolated from Protaetia brevitaris seulensis affected coagulation by inhibiting platelet aggregation, myristoylated alanine-rich C-kinase substrate (MARCKS) phosphorylation and reduced $\mathrm{Ca}^{2+}$ peak in platelets. Moreover, they were able to prolong aPTT and PT and to inhibit thrombin and FXa production ${ }^{(38)}$. Finally, two compounds (1, cyclo(L-Pro-L-Tyr) and 2, $\mathrm{N}$-acetyltyramine) extracted from Tenebrio molitor prolonged aPTT and reduced platelet aggregation induced by ADP, collagen or U46619, but not by thrombin. Moreover, FXa production and activity were inhibited, but not thrombin, trypsin, plasmin, activated protein $\mathrm{C}$ or tPA. These compounds also inhibited MARCKS phosphorylation and expression of P-selectin and PAC-1, and reduced $\mathrm{Ca}^{2+}$ peak in platelets ${ }^{(39)}$.

\section{Other activities in vitro}

Table 3 presents seven studies ${ }^{(19,29,30,34,40-42)}$ evaluating other in vitro effects for seven different insects. Proteins from Bombyx mori, Tenebrio molitor and Gryllus bimaculatus ${ }^{(40)}$, Alphitobius diaperinus ${ }^{(29)}$ and Gryllodes sigillatus ${ }^{(34,41)}$ were able to inhibit activity of angiotensin-converting enzyme (ACE). These proteins also showed potential anti-diabetic 
Table 1. In vitro antioxidant activity of edible insects

\begin{tabular}{|c|c|c|c|c|}
\hline Sample & Concentrations & Method & $\begin{array}{l}\text { Antioxidant } \\
\text { activity }\end{array}$ & References \\
\hline Acheta domesticus, Tenebrio molitor- $\mathrm{PH}$ & $\begin{array}{l}0.05-5.0 \mathrm{mg} / \\
\mathrm{ml}\end{array}$ & DPPH & + & Messina et al., 2019 ${ }^{(14)}$ \\
\hline $\begin{array}{l}\text { Acheta domesticus, Rhynchophorus ferrugineus, Tenebrio molitor, Zophobas } \\
\text { morio }\end{array}$ & - & ABTS, DPPH, FRAP, $\mathrm{Fe}^{2+}$ chelating activity & + & $\begin{array}{l}\text { Botella-Martinez et al., } \\
2020^{(25)}\end{array}$ \\
\hline \multirow{2}{*}{$\begin{array}{l}\text { Allomyrina dichotoma, Apis mellifera, Gryllus bimaculatus, Protaetia } \\
\text { brevitarsis, Teleogryllus emma, Tenebrio molitor }\end{array}$} & $500 \mu \mathrm{g} / \mathrm{ml}$ & ABTS, DPPH, FRAP & + & \multirow[t]{2}{*}{ Pyo et al., 2020(28) } \\
\hline & $200 \mu \mathrm{g} / \mathrm{ml}$ & Nitric scavenging activity & + & \\
\hline Alphitobius diaperinus - PH & - & ABTS, ORAC & + & Sousa et al., 2020(29) \\
\hline Acheta domesticus, Tenebrio molitor & $10 \mathrm{mg} / \mathrm{ml}$ & DPPH & + & $\begin{array}{l}\text { Navarro del Hierro et al., } \\
2020^{(30)}\end{array}$ \\
\hline Bombyx mori & $10 \mathrm{~g} / 50 \mathrm{ml}$ & DPPH, ABTS, FRAP & + & \multirow{3}{*}{$\begin{array}{l}\text { Anuduang et al., } 2020^{(31)} \\
\text { Dutta et al., 2017(32) }\end{array}$} \\
\hline \multirow[t]{2}{*}{ Brachytrupes orientalis } & $\begin{array}{l}0.25-6.25 \mathrm{mg} / \\
\mathrm{ml}\end{array}$ & $\mathrm{DPPH}$ & + & \\
\hline & $\begin{array}{l}1.25-12.5 \mathrm{mg} / \\
\mathrm{ml}\end{array}$ & FRAP, SAHR, SRSC & + & \\
\hline \multirow[t]{2}{*}{ Clanis bilineata } & $10-200 \mu \mathrm{g} / \mathrm{ml}$ & DPPH & + & \multirow[t]{2}{*}{ Sun et al., $2018^{(33)}$} \\
\hline & $0.1-4.0 \mathrm{mg} / \mathrm{ml}$ & $\beta$-carotene and linolenic acid bleaching test & + & \\
\hline Gryllodes sigillatus - PH & $1 \mathrm{mg} / \mathrm{ml}$ & $\begin{array}{l}\text { ABTS, DPPH, metal ion chelating activity } \\
\text { FRAP }\end{array}$ & + & Hall et al., $2018^{(34)}$ \\
\hline Gryllodes sigillatus, Schistocerca gregaria, Tenebrio molitor-PH & - & ABTS, DPPH, $\mathrm{Fe}^{2+}$ chelating activity & + & Zielinska et al., 2017 $7^{(15)}$ \\
\hline 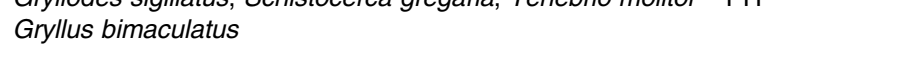 & - & $\begin{array}{l}\text { ABTS } \\
\text { DPPH }\end{array}$ & $\begin{array}{l}+ \\
+\end{array}$ & Hwang et al., $2019^{(6)}$ \\
\hline Hermetia illucens - PH & $14 \mathrm{~g} / \mathrm{l}$ & SAHR & + & Mintah et al., 2019a $a^{(17)}$ \\
\hline Hermetia illucens - PH & $\begin{array}{l}2 \mathrm{mg} / \mathrm{ml} \\
4 \mathrm{mg} / \mathrm{ml}\end{array}$ & $\begin{array}{l}\text { ABTS } \\
\text { FRAP, SRSC }\end{array}$ & $\begin{array}{l}+ \\
+\end{array}$ & Mintah et al., 2019b $\mathrm{b}^{(16)}$ \\
\hline Honey-bee brood & $0.0025 \mathrm{~g} / \mathrm{ml}$ & DPPH & + & Haber et al., 2019(18) \\
\hline Pachymerus nucleorum & $1 \mathrm{~g} / 100 \mathrm{ml}$ & ABTS & + & Alves et al., $2016^{(19)}$ \\
\hline Tenebrio molitor & - & ABTS, DPPH & + & Son et al., $2020^{(20)}$ \\
\hline Tenebrio molitor & $3 \mathrm{~g} / 10 \mathrm{ml}$ & ABTS, DPPH, FRAP & + & Mancini et al., 2019(21) \\
\hline Tenebrio molitor & $\begin{array}{l}0.625-5.0 \mathrm{mg} / \\
\mathrm{ml}\end{array}$ & $\begin{array}{l}\text { DPPH, FRAP, ORAC, SAHR, hydrogen peroxide radical } \\
\text { scavenging activity }\end{array}$ & + & Tang et al., 2018 \\
\hline Tenebrio molitor, Ulomoides dermestoides - $\mathrm{PH}$ & $0.1-1.0 \mathrm{mg} / \mathrm{ml}$ & ABTS & + & Flores et al., 2020(23) \\
\hline \multirow[t]{3}{*}{ Vespa affinis $\mathrm{L}$. } & $\begin{array}{l}0.25-6 \cdot 25 \mu \mathrm{g} / \\
\mu \mathrm{l}\end{array}$ & DPPH & + & \multirow{3}{*}{ Dutta et al., $2016^{(24)}$} \\
\hline & $\begin{array}{l}1 \cdot 25-15 \cdot 0 \mu \mathrm{g} / \\
\mu \mathrm{l}\end{array}$ & SAHR, SRSC & + & \\
\hline & $\begin{array}{l}1.25-10.0 \mu \mathrm{g} / \\
\mu \mathrm{l}\end{array}$ & Activities of CAT and GST enzymes & + & \\
\hline $\begin{array}{l}\text { Various (Acheta domesticus, Alphitobius diaperinus, Bombyx mori, } \\
\text { Calliptamus italicus, } \\
\text { Imbrasia oyemensis, Lasius niger, Lethocerus indicus, Rhynchophorus fer- } \\
\text { rugineus, } \\
\text { Scolopendra, Tanna japonensis, Tenebrio molitor, Haplopelma albostriatum, } \\
\text { Pandinus imperator) }\end{array}$ & - & ABTS hydro, ABTS lipo, FRAP & + & Di Mattia et al., 2019(26) \\
\hline $\begin{array}{l}\text { Various (Crocothemis servilia, Cybister tripunctatus, Hydrophilus olivaceous, } \\
\text { Laccotrephes maculatus, Lethocerus indicus) }\end{array}$ & $1-500 \mathrm{mg} / \mathrm{ml}$ & DPPH & + & Shantibala et al., $2014^{(27)}$ \\
\hline
\end{tabular}

PH, protein hydrolysates; DPPH, 2,2-diphenyl-1-picrylhydrazyl; ABTS, 2,2'-azino-bis(3-ethylbenzothiazoline-6-sulfonic acid; FRAP, ferric reducing antioxidant power; ORAC, oxygen radical absorbance capacity; SAHR, scavenging activity on hydroxyl radicals; RSC, superoxide radical scavenging capacity; CAT, catalase; GST, glutathione $S$-transferase. 
Table 2. In vitro anti-platelet aggregation activity of edible insects

\begin{tabular}{|c|c|c|c|c|}
\hline Sample & Concentrations & Activity & Result & References \\
\hline \multirow{4}{*}{$\begin{array}{l}\text { Allomyrina dichotoma, Apis mellifera, Gryllus bimaculatus, Protaetia brevitarsis, } \\
\text { Tenebrio molitor - WS }\end{array}$} & $5.0 \mathrm{mg} / \mathrm{ml}$ & Prolonged PT, TT, aPTT & - & \multirow{9}{*}{$\begin{array}{l}\text { Pyo et al., } \\
2020^{(28)}\end{array}$} \\
\hline & $0.25 \mathrm{mg} / \mathrm{ml}$ & Platelet aggregation & $\begin{array}{l}+ \\
(\mathrm{A}- \\
\mathrm{M}-)\end{array}$ & \\
\hline & $1.0 \mathrm{mg} / \mathrm{ml}$ & Haemolytic activity & - & \\
\hline & & & $\begin{array}{l}(A- \\
M+)\end{array}$ & \\
\hline \multirow[t]{5}{*}{ Teleogryllus emma - WS } & $5.0 \mathrm{mg} / \mathrm{ml}$ & Prolonged relative TT and aPTT & + & \\
\hline & $5.0 \mathrm{mg} / \mathrm{ml}$ & Prolonged PT & - & \\
\hline & $\begin{array}{l}5 \cdot 0,6 \cdot 0 \\
\quad 7.0 \mathrm{mg} / \mathrm{ml}\end{array}$ & Relative PTT, TT, aPTT & + & \\
\hline & $0.25 \mathrm{mg} / \mathrm{ml}$ & Platelet aggregation & + & \\
\hline & $1.0 \mathrm{mg} / \mathrm{ml}$ & Haemolytic activity & + & \\
\hline \multirow[t]{4}{*}{ Oxya chinensis sinuosa - 5 CPs ( $N$-acetyldopamine dimers) } & - & $\begin{array}{l}\text { Inhibition of thrombin, trypsin, elastase, plasmin, protein } \mathrm{Ca} \text {, streptoki- } \\
\text { nase, tPA and urokinase }\end{array}$ & - & \multirow{4}{*}{$\begin{array}{l}\text { Lee et al., } \\
2017 b^{(37)}\end{array}$} \\
\hline & $1,2,5 \mu \mathrm{M}$ & Reduction of platelet aggregation induced by ADP or U46619 & $+(5-)$ & \\
\hline & $1,2,5 \mu \mathrm{M}$ & Reduction of platelet aggregation induced by thrombin & - & \\
\hline & $2,5 \mu \mathrm{M}(\mathrm{c} 1-4)$ & Inhibition of FXa & + & \\
\hline \multirow[t]{5}{*}{ Protaetia brevitaris seulensis - 2 CPs (Indole alkaloids) } & $5-50 \mu \mathrm{M}$ & Inhibition of platelet aggregation induced by U46619 or collagen & + & \multirow{5}{*}{$\begin{array}{l}\text { Lee et al., } \\
\qquad 2017 a^{(38}\end{array}$} \\
\hline & $50 \mu \mathrm{M}$ & $\begin{array}{l}\text { Inhibition of ADP- and U46619-induced MARCKS phosphorylation (plate- } \\
\text { lets) }\end{array}$ & + & \\
\hline & $10,25,50 \mu \mathrm{M}$ & Reduction of $\mathrm{Ca}^{2+}$ peak (platelets) & + & \\
\hline & $5-50 \mu \mathrm{M}$ & Prolonged aPTT and PT & + & \\
\hline & Various & Inhibition of thrombin and FXa production & + & \\
\hline \multirow[t]{9}{*}{ Tenebrio molitor - 2 CPs (1, cyclo(L-Pro-L-Tyr) and 2, N-acetyltyramine) } & $1,5,10 \mu \mathrm{M}$ & Prolonged aPTT & + & \multirow{9}{*}{$\begin{array}{l}\text { Lee et al., } \\
\quad 2017 c^{(39)}\end{array}$} \\
\hline & $1,5,10 \mu \mathrm{M}$ & Reduction of platelet aggregation induced by ADP, collagen or U46619 & + & \\
\hline & $1,5,10 \mu \mathrm{M}$ & Reduction of platelet aggregation induced by thrombin & - & \\
\hline & $1-10 \mu \mathrm{M}$ & Inhibition of FXa production & + & \\
\hline & $5,10 \mu \mathrm{M}$ & Inhibition of FXa activity & + & \\
\hline & - & Inhibition of thrombin, trypsin, plasmin, activated protein C, and tPA & - & \\
\hline & $10 \mu \mathrm{M}$ & $\begin{array}{l}\text { Inhibition of ADP- and U46619-induced MARCKS phosphorylation (plate- } \\
\text { lets) }\end{array}$ & + & \\
\hline & $1,5,10 \mu \mathrm{M}$ & Reduction of $\mathrm{Ca}^{2+}$ peak (platelets) & + & \\
\hline & $5,10 \mu \mathrm{M}$ & $\begin{array}{l}\text { Reduction of ADP- or U46619-induced expression of P-selectin and PAC- } \\
1 \text { (platelets) }\end{array}$ & + & \\
\hline
\end{tabular}

WS, water-soluble extract; PT, prothrombin time; TT, thrombin time; aPTT, activated partial thromboplastin time; CP, compound; tPA, tissue plasminogen activator; ADP, adenosine diphosphate; FXa, factor Xa; MARCKS, myristoylated alanine-rich C-kinase substrate. 
activity by inhibition of dipeptidyl peptidase-4 (DPP-IV) ${ }^{(34)}$ and $\alpha$-glucosidase ${ }^{(40,41)}$. Moreover, extracts from Acheta domesticus and Tenebrio molitor were able to inhibit pancreatic lipase ${ }^{(30)}$, while Pachymerus nucleorum larvae showed tryptic activity together with absence of anti-nutritional factors as well as anti-tryptic and chymotryptic activity ${ }^{(19)}$. Lastly, flour from Tenebrio molitor affected the growth of Lactobacillus and Bifidobacterium, improving short-chain fatty acid (SCFA) production and viability in nutritive stress conditions ${ }^{(42)}$.

\section{Antioxidant activity in cellular models}

Nine studies ${ }^{(6,20,24,32,39,40,43-45)}$ investigating the antioxidant activity in cellular model of ten species of insects are described in Table 4. Gryllus bimaculatus and Tenebrio molitor were the most utilised insects, examined in three studies. Different fractions of the insects, including water-soluble fraction $(n=6)$, compounds $(n=2)$, protein hydrolysates $(n=1)$ and lipo-soluble fraction $(n=1)$, were tested. In three different studies, aqueous extracts of Gryllus bimaculatus ${ }^{(6)}$, methanolic extract of defatted powder and unsaponifiable lipids, obtained by Tenebrio molitor ${ }^{(20)}$, and Bombyx mori protein hydrolysates showed the ability to reduce lipopolysaccharide-induced nitric oxide (NO) production in the murine macrophage cell line RAW 264.7. A similar effect was exerted by 1, cyclo(L-Pro-LTyr) and 2, $N$-acetyltyramine isolated from Tenebrio molitor in human umbilical vein endothelial cells (HUVECs) ${ }^{(39)}$ and by glycosaminoglycan from Gryllus bimaculatus in diabetic type 2 microvascular endothelial cells (D-HMVECs) ${ }^{(45)}$. According to a study by Yoon and co-workers ${ }^{(40)}$, protein hydrolysates of Tenebrio molitor and Gryllus bimaculatus did not exert any effect on NO release. Water-soluble extract of dung beetles of Onitis sp., mole crickets of Gryllotalpa sp., grasshopper of Caelifera sp. ${ }^{(43)}$, Oryctes boas and Zonocerus variegatus ${ }^{(44)}$ were tested in human peripheral blood lymphocytes to evaluate whether they affected oxidative status. Results showed that at lower concentrations (10-40 ppm) the insects display an antioxidant effect; however, at higher concentrations (2000 ppm) they exhibited a pro-oxidant effect. According to the results obtained in a cell-free system ${ }^{(24)}$, the aqueous extract of Vespa affinis was able to increase the activity of both GST and CAT also in THP-1 human monocytes and human plasma; moreover, it reduced reactive oxygen species (ROS) formation in THP-1. Finally, the hydro-alcoholic extract of Brachytrupes oriental was able to restore nuclear factor erythroid 2-related factor (Nrf2) and GST protein expression, reducing radical and malondialdehyde (MDA) levels in $\mathrm{C} 2 \mathrm{C} 12$, a murine myotube cell line, following high glucose stress ${ }^{(32)}$.

\section{Other activity in cellular models or ex vivo}

The effect of edible insects on coagulation markers ${ }^{(37-39)}$, inflammatory status ${ }^{(41,46)}$ and lipid metabolism ${ }^{(7,47-49)}$ are described in Table 5. A total of eleven studies involving eight insects were reported: Protaetia brevitaris was cited in three research articles, whilst Oxya chinensis, Gryllus bimaculatus and Tenebrio molitor were investigated in two studies each. Compounds (indole alkaloids) isolated from Protaetia brevitaris seulensis were able to reduce prothrombin-produced thrombin, plasminogen 
Table 4. Antioxidant activity of edible insects in cellular models or ex vivo

\begin{tabular}{|c|c|c|c|c|}
\hline Sample & Cell type & Concentration & Antioxidant/Oxidant Marker & References \\
\hline Bombyx mori-PH & RAW264.7 & $0.1,0.3,0.5 \mathrm{mg} / \mathrm{ml}$ & $\mathrm{NO} \downarrow$ & Yoon et al., $2019^{(40)}$ \\
\hline Gryllus bimaculatus - $\mathrm{PH}$ & & & $\mathrm{NO} \leftrightarrow$ & \\
\hline Tenebrio molitor- $\mathrm{PH}$ & & & $\mathrm{NO} \leftrightarrow$ & \\
\hline Brachytrupes orientalis - WS & $\mathrm{C} 2 \mathrm{C} 12$ & $7.5,10 *, 12.5 * \mathrm{mg} / \mathrm{ml}$ & Lipid peroxidation: MDA $\downarrow^{*} ; \mathrm{ROS} \downarrow^{*}$; GST, Nrf-2 $\uparrow^{*}$ & Dutta et al., 2017(32) \\
\hline Caelifera sp. - WS & hPBL & $5-2000$ ppm & $\begin{array}{l}\text { TOS } \leftrightarrow(\uparrow 2000 \mathrm{ppm}) \\
\text { TAC } \leftrightarrow(\downarrow 2000 \mathrm{ppm})\end{array}$ & Koc et al., 2014 ${ }^{(43)}$ \\
\hline Gryllotalpa sp. - WS & & & $\begin{array}{l}\text { TOS } \leftrightarrow(\uparrow 2000 \mathrm{ppm}) ; \\
\text { TAC } \leftrightarrow(\uparrow 10 \mathrm{ppm}, \downarrow 1000,2000 \mathrm{ppm})\end{array}$ & \\
\hline Onitis sp. - WS & & & $\begin{array}{l}\text { TOS } \leftrightarrow(\uparrow 2000 \mathrm{ppm}) \\
\text { TAC } \leftrightarrow(\uparrow 15 \mathrm{ppm}, \downarrow 1000,2000 \mathrm{ppm})\end{array}$ & \\
\hline Gryllus bimaculatus - CP (glycosaminoglycan) & D-HMVECs & $5,10 \mathrm{mg} / \mathrm{ml}$ & NO $\downarrow$ & Ahn et al., 2020(45) \\
\hline Gryllus bimaculatus - WS & RAW264.7 & $20-100 \mu \mathrm{g} / \mathrm{ml}$ & $\mathrm{NO} \downarrow$ & Hwang et al., 2019(6) \\
\hline Oryctes boas - WS & hPBL & $5-2000$ ppm & $\begin{array}{l}\text { TAC } \uparrow(10-40 \mathrm{ppm}), \downarrow(2000 \mathrm{ppm}) \\
\text { TOS } \uparrow(1000,2000 \mathrm{ppm})\end{array}$ & Memis et al., $2013^{(44)}$ \\
\hline Zonocerus variegatus - WS & & & $\begin{array}{l}\text { TAC } \uparrow(10-25 \mathrm{ppm}), \downarrow(500-2000 \mathrm{ppm}) \\
\text { TOS } \uparrow(200-2000 \mathrm{ppm})\end{array}$ & \\
\hline Tenebrio molitor - $2 \mathrm{CPs}$ & HUVECs & $5,10 \mu \mathrm{M}$ & NO $\downarrow$ & Lee et al., 2017c(39) \\
\hline Tenebrio molitor - WS, LS & RAW264.7 & $\begin{array}{l}\text { WS: } 25-500 \mu \mathrm{g} / \mathrm{ml} \\
\text { LS: } 0.05-5.0 \mu \mathrm{g} / \mathrm{ml}\end{array}$ & NO $\downarrow$ & Son et al., $2020^{(20)}$ \\
\hline \multirow[t]{3}{*}{ Vespa affinis - WS } & THP-1 & $0.4,0.8 *, 1 \cdot 2 * \mu \mathrm{g} / \mu \mathrm{l}$ & GST, CAT $\uparrow^{*}$ & Dutta et al., 2016(24) \\
\hline & THP-1 & $0.8 \mu \mathrm{g} / \mu \mathrm{l}$ & ROS $\downarrow$ & \\
\hline & hPlasma & $1.25-10.00 \mu \mathrm{g} / \mu \mathrm{l}$ & $\begin{array}{l}\text { GST } \uparrow \text { (except for } 1.25 \text { and } 2.50 \mu \mathrm{g} / \mu \mathrm{l}) \\
\text { CAT } \uparrow \text { (except for } 1.25 \mu \mathrm{g} / \mu \mathrm{l})\end{array}$ & \\
\hline
\end{tabular}

$\mathrm{PH}$, protein hydrolysates; NO, nitric oxide; WS, water-soluble extract; MDA, malondialdehyde; ROS, reactive oxygen species; GST, glutathione S-transferase; Nrf2, nuclear factor erythroid 2-related factor; TOS, total oxidant status; TAC, total antioxidant capacity; CP, compound; D-HMVECs, diabetic type 2 microvascular endothelial cells; hPBL, human peripheral blood lymphocytes; HUVECs, human umbilical vein endothelial cells; LS, lipo-soluble extract; CAT, catalase. 
Table 5. Other activity in cellular models or ex vivo

\begin{tabular}{|c|c|c|c|c|}
\hline Source & Cells & Dose & Results & References \\
\hline \multirow[t]{2}{*}{ Allomyrina dichotoma - CPs (Tetrahydroquinolines) } & HUVECS & $\begin{array}{l}1,2,5^{*}, 10^{*} \\
\mu \mathrm{M}\end{array}$ & $\begin{array}{l}\text { Cell viability } \leftrightarrow \text {; permeability } \downarrow *, * * \text {; VCAM- } 1 *, * *, \text { ICAM- } 1 * * \downarrow \text {; adherence of monocytes to } \\
\text { HUVEC } \\
\text { monolayers } \downarrow * * * \text {; migration of human neutrophils through HUVEC monolayers } \downarrow^{*}, * *\end{array}$ & $\begin{array}{l}\text { Park et al., } \\
2020^{(46)}\end{array}$ \\
\hline & HUVECs & $10 \mu \mathrm{M}$ & NF-kB p65 activity; TNF- $\alpha$ and IL-1 $\beta$ production, expression of phospho-p38 $\downarrow$ & \\
\hline \multirow[t]{2}{*}{ Bombyx mori - PUFAs } & $\mathrm{L}-02$ & $\begin{array}{l}100-1600 \mu \mathrm{g} / \\
\mathrm{ml}\end{array}$ & Cell viability $\leftrightarrow(\downarrow 1600 \mu \mathrm{g} / \mathrm{ml})$ & $\begin{array}{l}\text { Luo et al., } \\
2020^{(7)}\end{array}$ \\
\hline & & $\begin{array}{l}200,400 * \\
800 * * \mu \mathrm{g} / \\
\mathrm{ml}\end{array}$ & $\begin{array}{l}\text { Lipid accumulation, TC } \downarrow * * * * \text {;BA } \uparrow ; \text { mRNA and protein expression (cholesterol metabolism } \\
\text { related): LXR } \alpha * *, \text { PPAR } \gamma *, * *, A B C A 1 *, * *, A B C G 1, \text { CYP7A } 1 * * * \uparrow\end{array}$ & \\
\hline \multirow[t]{2}{*}{ Bombyx mori - $\alpha$-linolenic acid } & & $\begin{array}{l}2 \cdot 5-40 * \mu \mathrm{g} / \\
\mathrm{ml}\end{array}$ & Cell viability: $\leftrightarrow(\downarrow 40 \mu \mathrm{g} / \mathrm{mL})$ & \\
\hline & & $\begin{array}{l}5,10 *, 20 * * \\
\mu \mathrm{g} / \mathrm{ml}\end{array}$ & $\begin{array}{l}\text { Lipid accumulation, TC } \downarrow^{*}, * * ; \text { TBA } \uparrow ; \text { mRNA and protein expression (chol-metabolism related): } \\
\text { LXR } \alpha, \text { PPAR } \gamma, \text { ABCA1, ABCG1, CYP7A1 }{ }^{* *}\end{array}$ & \\
\hline Gryllodes sigillatus - PH & RAW 264.7 & $0.5-3.0 \mu \mathrm{g} / \mathrm{ml}$ & Viability $\leftrightarrow ; N F-k B \downarrow$ & $\begin{array}{l}\text { Hall et al., } \\
2020^{(41)}\end{array}$ \\
\hline Gryllus bimaculatus - CP (glycosaminoglycan) & D- HMVECs & $5,10 * \mathrm{mg} / \mathrm{ml}$ & Laminin $\leftrightarrow$; VEGF $\uparrow^{*}$ & $\begin{array}{l}\text { Ahn et al., } \\
2020^{(45)}\end{array}$ \\
\hline $\begin{array}{l}\text { Gryllus bimaculatus, Oxya chinensis sinuosa, Protaetia } \\
\text { brevitarsis seulensis - WS }\end{array}$ & HepG2 & $\begin{array}{l}10-200 \mu \mathrm{g} / \mathrm{ml} \\
10,50 \\
100 \mu \mathrm{g} / \mathrm{ml}\end{array}$ & $\begin{array}{l}\text { Cell viability } \leftrightarrow \\
\text { Intracellular lipid accumulation, TG } \downarrow\end{array}$ & $\begin{array}{l}\text { Im et al., } \\
2018^{(47)}\end{array}$ \\
\hline Locusta migratoria - WS & hPBL & $1-1000 \mathrm{mg} / \mathrm{l}$ & MN/1000 cells, SCEs, CAs $\leftrightarrow$ & $\begin{array}{l}\text { Tukez et al., } \\
\quad 2014^{(50)}\end{array}$ \\
\hline $\begin{array}{l}\text { Oxya chinensis sinuosa - } 5 \mathrm{CPs} \text { ( } \mathrm{N} \text {-acetyldopamine } \\
\text { dimers) }\end{array}$ & Mice plasma & $1,2,5 \mu \mathrm{M}$ & aPTT $;$ PT $\leftrightarrow$ & $\begin{array}{l}\text { Lee et al., } \\
\qquad 2017 b^{(37)}\end{array}$ \\
\hline $\begin{array}{l}\text { Oxya chinensis sinuosa - } 1-4 \mathrm{CPs} \text { ( } \mathrm{N} \text {-acetyldopamine } \\
\text { dimers) }\end{array}$ & HUVECs & $0.5-5 \cdot 0 \mu \mathrm{M}$ & FXa $\downarrow$ (except for $0.5 \mu \mathrm{M})$ & \\
\hline \multirow[t]{2}{*}{$\begin{array}{l}\text { Protaetia brevitaris seulensis }-5 \mathrm{CPs} \text { (results are } \\
\quad \text { referred to CPs } 1 \text { and } 2 \text { ) (Indole alkaloids) }\end{array}$} & HUVECs & $\begin{array}{l}5-50 \mu \mathrm{M} \\
0.5-5.0 \mu \mathrm{M}\end{array}$ & $\begin{array}{l}\text { Prothrombin-produced thrombin, PAI } 1 \downarrow \text { (except for cp5) tPA } \leftrightarrow \\
\text { FXa } \downarrow \text { (except for } 0.5 \mu \mathrm{M} \text { ) }\end{array}$ & $\begin{array}{l}\text { Lee et al., } \\
\qquad 2017 a^{(38)}\end{array}$ \\
\hline & $\begin{array}{l}\text { Mice platelet- } \\
\text { rich plasma }\end{array}$ & $10,25,50 \mu \mathrm{M}$ & Aggregation \% $\downarrow$; aPTT, PT $\uparrow$ & \\
\hline Protaetia brevitarsis - WS & 3T3-L1 & $\begin{array}{l}0.1,0.5 * \\
1.0 * \mathrm{mg} / \mathrm{ml}\end{array}$ & Adipogenesis-related genes (mRNA expression): C/EBP $\alpha$, aP2, FAS $\downarrow *$ & $\begin{array}{l}\text { Ahn et al., } \\
2019^{(49)}\end{array}$ \\
\hline $\begin{array}{l}\text { Tenebrio molitor - } 2 \text { CPs (1, cyclo(L-Pro-L-Tyr) and } 2 \text {, } \\
\quad N \text {-acetyltyramine) }\end{array}$ & $\begin{array}{l}\text { Mice blood } \\
\text { HUVECs }\end{array}$ & $\begin{array}{l}1,5,10 \mu \mathrm{M} \\
5,10 \mu \mathrm{M}\end{array}$ & $\begin{array}{l}\text { aPTT, clotting time } \uparrow \\
\mathrm{ET}-1 \uparrow\end{array}$ & $\begin{array}{l}\text { Lee et al., } \\
\qquad 2017 c^{(39)}\end{array}$ \\
\hline Tenebrio molitor & 3T3-L1 & $\begin{array}{l}0 \cdot 1,1^{*}, 2^{*} \\
\quad 3^{*} \mathrm{mg} / \mathrm{ml}\end{array}$ & $\begin{array}{l}\text { Lipid droplet formation, TG } \downarrow^{*} \text {; adipogenic differentiation (mRNA expression): PPAR } \gamma \text {, } \\
\text { C/EBP- } \alpha \downarrow^{*} \text {; lipogenesis-specific genes mRNA expression: SREBP-1c, LPL, SCD1, } \\
\text { FAS } \downarrow *(\text { RRNA); p-ERK } \downarrow \text {, p-AMPK- } \alpha, p-p 38 \uparrow, p-J N K \leftrightarrow\end{array}$ & $\begin{array}{l}\text { Seo et al., } \\
2017^{(48)}\end{array}$ \\
\hline
\end{tabular}

HUVECs, human umbilical vein endothelial cells; VCAM-1, vascular cell adhesion molecule-1; ICAM-1, intercellular adhesion molecule-1; NF-kB, nuclear factor-kB; TNF- $\alpha$, tumour necrosis factor- $\alpha$; LL-1 $\beta$, interleukin-1 $1 \beta$; PUFA, polyunsaturated fatty acids; TC, total cholesterol; TBA, total bile acid; LXR $\alpha$, liver X receptor; PPAR $\gamma$, peroxisome proliferator-activated receptor $\gamma$; ABCA1, ATP-binding cassette transporter A1; ABCG1, ATP-binding cassette transporter G1; CYP7A1, cholesterol 7 $\alpha$ hydroxylase; PH, protein hydrolysates; D-HMVECs, diabetic type 2 microvascular endothelial cells; VEGF, vascular endothelial growth factor; WS, water-soluble extract; TG, triacylglycerol; MN, micronucleus; SCE, sister chromatid exchange; CA, chromosome aberration; aPTT, activated partial thromboplastin time; PT, prothrombin time; FXa, factor Xa; PAl-1, plasminogen activator inhibitor-1; tPA, tissue plasminogen activator; C/EBP $\alpha$, CCAAT/ enhancer-binding protein $\alpha$; FAS, fatty acid synthase; ET-1, endothelin-1; SREBP-1C, sterol regulatory element-binding protein 1C; LPL, lipoprotein lipase; SCD1, stearoyl-CoA desaturase-1; ERK, extracellular signal-regulated kinases; AMPK- $\alpha$, adenosine monophosphate-activated protein kinase- $\alpha$; JNK, c-Jun $N$-terminal kinase. 
Table 6. Effect of edible insects on body weight and composition, inflammation, redox, lipid and glycaemia/insulin status and coagulation markers in animal and human studies

\begin{tabular}{|c|c|c|c|c|c|c|c|c|c|c|}
\hline \multirow[b]{2}{*}{ Sample } & \multirow[b]{2}{*}{ Animal/disease } & \multirow[b]{2}{*}{ Dose } & \multirow[b]{2}{*}{ Duration } & \multicolumn{6}{|c|}{ Results } & \multirow[b]{2}{*}{ References } \\
\hline & & & & $\begin{array}{l}\text { Body and organ } \\
\text { weight and compo- } \\
\text { sition }\end{array}$ & $\begin{array}{l}\text { Inflammatory } \\
\text { status }\end{array}$ & $\begin{array}{l}\text { Antioxidant/ } \\
\text { oxidant } \\
\text { markers }\end{array}$ & Lipid status & $\begin{array}{l}\text { Glycaemia/ } \\
\text { insulin status }\end{array}$ & $\begin{array}{l}\text { Coagulation } \\
\text { markers }\end{array}$ & \\
\hline \multirow[t]{4}{*}{ Acheta domesticus } & $\begin{array}{l}\text { Malnourished wean- } \\
\text { ling Sprague- } \\
\text { Dawley rats }\end{array}$ & $283.0 \mathrm{~g} / \mathrm{kg}$ & $14 \mathrm{~d}$ & $\begin{array}{l}\text { Body weight gain, } \\
\text { organs weight } \uparrow\end{array}$ & & & & & & $\begin{array}{l}\text { Agbemafle } \\
\text { et al., } \\
2019^{(61)}\end{array}$ \\
\hline & & & & $\begin{array}{l}\text { Relative biological } \\
\text { value } \uparrow \\
\text { Bone mineral con- } \\
\quad \text { tent, lean mass, } \uparrow \\
\text { Fat mass } \uparrow\end{array}$ & & & & & & \\
\hline & $\begin{array}{l}\text { Malnourished wean- } \\
\text { ling Sprague- } \\
\text { Dawley rats }\end{array}$ & $154.3 \mathrm{~g} / \mathrm{kg}$ & $14 d$ & $\begin{array}{c}\text { Body weight gain, } \\
\text { organ weight } \uparrow\end{array}$ & & & & & & \\
\hline & & & & $\begin{array}{l}\text { Relative biological } \\
\text { value } \leftrightarrow \\
\text { Bone mineral con- } \\
\text { tent, lean mass } \uparrow \\
\text { Fat mass } \leftrightarrow\end{array}$ & & & & & & \\
\hline $\begin{array}{l}\text { Rhynchophorus phoe- } \\
\text { nicis fabricius }\end{array}$ & $\begin{array}{l}\text { Malnourished wean- } \\
\text { ling Sprague- } \\
\text { Dawley rats }\end{array}$ & $403.0 \mathrm{~g} / \mathrm{kg}$ & $14 \mathrm{~d}$ & $\begin{array}{l}\text { Body weight gain, } \\
\text { organ weight } \uparrow \\
\text { Relative biological } \\
\text { value } \uparrow \\
\text { Bone mineral con- } \\
\text { tent, lean mass } \uparrow \\
\text { Fat mass } \leftrightarrow\end{array}$ & & & & & & \\
\hline \multirow[t]{2}{*}{$\begin{array}{l}\text { Allomyrina dichotoma } \\
-4 \text { CPs (results are } \\
\text { referred to as CPs } \\
1,2 \text { and } 3 \text { ) } \\
\text { (Tetrahydroquinolin- } \\
\text { es) }\end{array}$} & $\begin{array}{l}\text { C57BL/6 mice, LPS } \\
\text { inflammation }\end{array}$ & $\begin{array}{l}0.13 \\
0.26 * \\
\mu \mathrm{M} \text { per } \\
\text { mouse }\end{array}$ & Acute & & $\begin{array}{l}\text { Peritoneum: } \\
\text { leucocyte } \\
\text { migration, } \\
\text { vascular per- } \\
\text { meability } \downarrow *\end{array}$ & & & & & $\begin{array}{l}\text { Park et al., } \\
2020^{(46)}\end{array}$ \\
\hline & $\begin{array}{l}\text { CLP-induced septic } \\
\text { C57BL/6 mice }\end{array}$ & $\begin{array}{l}0.26 \mu \mathrm{M} \\
\text { per } \\
\text { mouse }\end{array}$ & Acute & Survival rate $\uparrow$ & $\begin{array}{l}\text { Lungs: intersti- } \\
\text { tial oedema, } \\
\text { tissue dam- } \\
\text { age } \leftrightarrow\end{array}$ & & & & & \\
\hline Bombyx mori & $\begin{array}{l}\text { Sprague-Dawley rats, } \\
\text { healthy }\end{array}$ & $200 \mathrm{~g} / \mathrm{kg}$ & 5 weeks & Body weight $\leftrightarrow$ & & & TG, TC $\downarrow ; \mathrm{HDL} \leftrightarrow$ & & & Ryu, 2014(56) \\
\hline Bombyx mori - LS & $\begin{array}{l}\text { Wistar rats, hypercho- } \\
\text { lesterolaemia }\end{array}$ & $\begin{array}{l}1^{*}, 2^{* *} \\
4^{* * *} \\
\mathrm{~mL} / \mathrm{kg} / \mathrm{d}\end{array}$ & 6 weeks & $\begin{array}{l}\text { Organ size: liver*** } \\
\quad \downarrow ; \text { kidney } \downarrow ; \\
\text { heart* } \downarrow ; \\
\text { spleen** } \uparrow\end{array}$ & & $\begin{array}{l}\text { Serum: } \\
\text { TAC**, } \\
\text { SOD } * *, \\
* * *, \text { GPx } \\
* *, * * \uparrow ; \\
\text { MDA } \downarrow \\
\text { Liver: TAC, } \\
\text { SOD } \uparrow ; \\
\text { GPx } \leftrightarrow ; \\
\text { MDA } \downarrow\end{array}$ & $\begin{array}{l}\text { TC, LDL, LDL/HDL } \downarrow ; \\
\text { TG, HDL }\end{array}$ & & & $\begin{array}{l}\text { Zou et al., } \\
2017^{(64)}\end{array}$ \\
\hline
\end{tabular}


Table 6. (Continued)

\begin{tabular}{|c|c|c|c|c|c|c|c|c|c|c|}
\hline \multicolumn{11}{|l|}{ Animal studies } \\
\hline \multirow[b]{2}{*}{ Sample } & \multirow[b]{2}{*}{ Animal/disease } & \multirow[b]{2}{*}{ Dose } & \multirow[b]{2}{*}{ Duration } & \multicolumn{6}{|c|}{ Results } & \multirow[b]{2}{*}{ References } \\
\hline & & & & $\begin{array}{l}\text { Body and organ } \\
\text { weight and compo- } \\
\text { sition }\end{array}$ & $\begin{array}{l}\text { Inflammatory } \\
\text { status }\end{array}$ & $\begin{array}{l}\text { Antioxidant/ } \\
\text { oxidant } \\
\text { markers }\end{array}$ & Lipid status & $\begin{array}{l}\text { Glycaemia/ } \\
\text { insulin status }\end{array}$ & $\begin{array}{l}\text { Coagulation } \\
\text { markers }\end{array}$ & \\
\hline $\begin{array}{l}\text { Clanis bilineata - CPs } \\
\text { (chitooligosacchar- } \\
\text { ides) }\end{array}$ & $\begin{array}{l}\text { Sprague-Dawley rats, } \\
\text { high-fat diet }\end{array}$ & $5 \%(w / w)$ & 6 weeks & $\begin{array}{l}\text { Body weight gain, } \\
\text { food efficiency } \\
\text { ratio } \downarrow \text {; food } \\
\text { intake } \leftrightarrow\end{array}$ & & & $\begin{array}{l}\text { TG, TC, LDL } \downarrow ; \mathrm{HDL} \uparrow \\
\text { Faecal content: TG, TC } \\
\quad \uparrow\end{array}$ & & & $\begin{array}{l}\text { Xia et al., } \\
2013^{(63)}\end{array}$ \\
\hline \multirow[t]{3}{*}{ Caelifera sp. } & Wistar rats, healthy & $\begin{array}{l}10,20 \mathrm{~g} / \\
\mathrm{kg}\end{array}$ & Acute & $\begin{array}{l}\text { Body weight } \leftrightarrow ; \text { tox- } \\
\text { icity signs: no; } \\
\text { mortality: no }\end{array}$ & & & & & & $\begin{array}{l}\text { Ochiai et al., } \\
2020^{(57)}\end{array}$ \\
\hline & & $1 *, 3 * * \%$ & $28 / 90 d$ & $\begin{array}{l}\text { Organs and tissues } \\
\text { weight } \leftrightarrow\end{array}$ & & & TG, TC, HDL ↔ & $\begin{array}{l}\text { Insulin } \downarrow \text { (**, } \\
90 \mathrm{~d}) \text {; } \\
\text { plasma glu- } \\
\text { cose } \uparrow^{*}, \downarrow^{* *} \\
(28 \mathrm{~d})\end{array}$ & & \\
\hline & & & & & & & $\begin{array}{l}\text { Liver: TG, TC } \leftrightarrow(28 \mathrm{~d}), \\
\uparrow(90 \mathrm{~d}) \\
\text { Caecum: faecal crude } \\
\text { lipids } \leftrightarrow(28 \mathrm{~d}), \uparrow(90 \\
\text { d); lipid excretion } \leftrightarrow \\
\left(\uparrow^{*} 90 \mathrm{~d}\right) ; \mathrm{TG}, \mathrm{TC} \leftrightarrow \\
\text { SCFA } \downarrow^{*}, \mathrm{pH} \leftrightarrow\end{array}$ & & & \\
\hline \multirow[t]{2}{*}{ Grain larvae - WS } & $\begin{array}{l}\text { Sprague-Dawley rats, } \\
\text { healthy }\end{array}$ & $\begin{array}{l}5 \cdot 0,7 \cdot 0 \\
9.0 \mathrm{mg}\end{array}$ & 4 weeks & $\begin{array}{l}\text { Body weight gain } \\
\leftrightarrow ; \text { weight: liver, } \\
\text { kidney } \leftrightarrow \text {; thy- } \\
\text { mus, spleen } \uparrow ; \\
\text { abdominal fat } \downarrow\end{array}$ & $\lg G, \lg A, \lg M \downarrow$ & & TG, TC, LDL $\downarrow$ & $\begin{array}{l}\text { Blood glucose } \\
\quad \downarrow\end{array}$ & & $\begin{array}{l}\text { Park \& Park } \\
2015^{(58)}\end{array}$ \\
\hline & & & & & & & $\begin{array}{l}\text { Caecal organic acids: } \\
\text { total, acetic, propi- } \\
\text { onic } \uparrow \text {; butyric, isobu- } \\
\text { tyric, valeric, } \\
\text { isovaleric } \downarrow\end{array}$ & & & \\
\hline \multirow[t]{3}{*}{$\begin{array}{c}\text { Green cocoon shell of } \\
\text { Bombyx mori-WS }\end{array}$} & $\begin{array}{l}\text { ICR mice, type } 2 \text { dia- } \\
\text { betes }\end{array}$ & $\begin{array}{c}150,250 * \\
350 * * \\
\mathrm{mg} / \mathrm{kg}\end{array}$ & 7 weeks & Body weight $\leftrightarrow$ & $\begin{array}{l}\text { Liver: NF-kB, } \\
\text { IL6, TNF- } \alpha \\
\downarrow^{*}, * *\end{array}$ & $\begin{array}{l}\text { Liver: GPx, } \\
\text { SOD } \uparrow ; \\
\text { MDA, 8- } \\
\text { OHdG } \downarrow^{*}, \\
* *\end{array}$ & $\begin{array}{l}\text { TC, HDL ↔; TG** } \downarrow ; \\
\text { LDL*,** } \downarrow\end{array}$ & $\begin{array}{l}\text { OGTT: blood } \\
\text { glucose } \\
\text { reduction } \\
\text { rate } \uparrow, \text { AUC } \\
\downarrow\end{array}$ & & $\begin{array}{l}\text { Zhao et al., } \\
2019^{(8)}\end{array}$ \\
\hline & & & & & $\begin{array}{l}\text { Liver: inflamma- } \\
\text { tory infiltra- } \\
\text { tions, } \\
\text { oedema } \downarrow\end{array}$ & & $\begin{array}{l}\text { Liver: lipid droplet accu- } \\
\text { mulation } \downarrow\end{array}$ & $\begin{array}{l}\text { ITT: blood glu- } \\
\text { cose } \downarrow \\
\text { AUC } \downarrow\end{array}$ & & \\
\hline & & & & & & & & $\begin{array}{l}\text { Fasting blood } \\
\text { glucose } \downarrow ; \\
\text { fasting } \\
\text { blood insulin } \\
\downarrow \text {; HbA1c*, } \\
\quad * *, \text { HOMA- } \\
\text { IR } \downarrow ; \text { ISI } \uparrow\end{array}$ & & \\
\hline
\end{tabular}


Table 6. (Continued)

\begin{tabular}{|c|c|c|c|c|c|c|c|c|c|c|}
\hline \multicolumn{11}{|l|}{ Animal studies } \\
\hline \multirow[b]{2}{*}{ Sample } & \multirow[b]{2}{*}{ Animal/disease } & \multirow[b]{2}{*}{ Dose } & \multirow[b]{2}{*}{ Duration } & \multicolumn{6}{|c|}{ Results } & \multirow[b]{2}{*}{ References } \\
\hline & & & & $\begin{array}{l}\text { Body and organ } \\
\text { weight and compo- } \\
\text { sition }\end{array}$ & $\begin{array}{l}\text { Inflammatory } \\
\text { status }\end{array}$ & $\begin{array}{l}\text { Antioxidant/ } \\
\text { oxidant } \\
\text { markers }\end{array}$ & Lipid status & $\begin{array}{l}\text { Glycaemia/ } \\
\text { insulin status }\end{array}$ & $\begin{array}{l}\text { Coagulation } \\
\text { markers }\end{array}$ & \\
\hline & & & & & & & & $\begin{array}{l}\text { Pancreas: islet } \\
\text { structure } \\
\text { and area, } \\
\text { pancreatic } \beta \\
\text { cells, insulin } \\
\text { secreted by } \\
\beta \text { cells } \uparrow\end{array}$ & & \\
\hline \multirow[t]{2}{*}{ Gryllodes sigillatus } & Mice, malnourished & - & 6 weeks & $\begin{array}{l}\text { Body weight } \uparrow \text { (ver- } \\
\text { sus malnour- } \\
\text { ished) }\end{array}$ & TLR4, TNF $\alpha$ & & $\begin{array}{l}\text { TG } \downarrow \text {; leptin, adiponec- } \\
\text { tin } \leftrightarrow \text { (versus control) }\end{array}$ & & & $\begin{array}{l}\text { Bergmans } \\
\quad \text { et a- } \\
\text { I,.2020(62) }\end{array}$ \\
\hline & & & & & $\begin{array}{l}\text { IL-1 } \beta, \text { IFN } \gamma, \text { IL- } \\
4 \text { in spleen } \\
\text { tissue } \leftrightarrow \\
\text { (versus con- } \\
\text { trol) }\end{array}$ & & & & & \\
\hline \multirow[t]{2}{*}{ Gryllus bimaculatus } & $\begin{array}{l}\text { Sprague-Dawley rats, } \\
\text { varicocele }\end{array}$ & $\begin{array}{l}1.63 \\
6.5 \mathrm{mg} / \\
\mathrm{kg}\end{array}$ & $42 d$ & $\begin{array}{l}\text { Body and organ } \\
\text { weights } \leftrightarrow\end{array}$ & TNF- $\alpha$, IL-6 $\downarrow$ & $\begin{array}{l}\text { Testicular } \\
\text { tissues }\end{array}$ & & & & $\begin{array}{l}\text { Karna et al., } \\
\text { 2020(52) }\end{array}$ \\
\hline & & & & & & $\begin{array}{l}\text { MDA, ROS/ } \\
\text { RNS } \downarrow \\
\text { SOD, GPx, } \\
\text { CAT } \uparrow\end{array}$ & & & & \\
\hline $\begin{array}{l}\text { Gryllus bimaculatus - } \\
\text { WS }\end{array}$ & Wistar rats, obesity & $\begin{array}{l}100 * \\
200 * * \\
\mathrm{mg} / \mathrm{kg}\end{array}$ & 2 months & $\begin{array}{l}\text { Body weight } \leftrightarrow \text {; fat } \\
\text { weight: abdomi- } \\
\text { nal**, total } \downarrow \text {, epi- } \\
\text { didymal } \leftrightarrow ; \\
\text { death/toxicity: no }\end{array}$ & 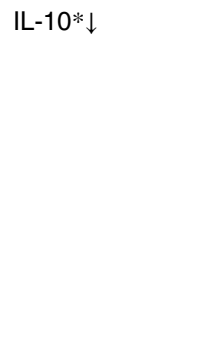 & $\begin{array}{l}\text { Blood pro- } \\
\text { tein car- } \\
\text { bonyl con- } \\
\text { tent } \\
\downarrow * *(2 \mathrm{~m}) \\
\text { CAT } \leftrightarrow \\
\text { Liver: MDA } \downarrow \\
\text { Serum: uric } \\
\text { acid } \leftrightarrow \downarrow \\
(1 \mathrm{~m}), \uparrow \\
(2 \mathrm{~m})\end{array}$ & $\begin{array}{l}\text { TG } \downarrow(100 \mathrm{mg} / \mathrm{kg}-1 \mathrm{~m}) ; \\
\text { TC, HDL, LDL } \leftrightarrow ; \\
\text { abdominal fat composi- } \\
\text { tion: polyunsaturated } \\
\uparrow, \text { monounsaturated } \\
\downarrow\end{array}$ & $\begin{array}{l}\text { Glucose } \downarrow(1 \mathrm{~m} \\
*), \leftrightarrow(2 \mathrm{~m})\end{array}$ & & $\begin{array}{l}\text { Ahn et al., } \\
2015^{(51)}\end{array}$ \\
\hline $\begin{array}{l}\text { Gryllus bimaculatus - } \\
\text { WS }\end{array}$ & $\begin{array}{l}\text { C57BL/6J mice, alco- } \\
\text { holic liver damage }\end{array}$ & $200 \mathrm{mg} / \mathrm{kg}$ & 2 weeks & & $\begin{array}{c}\mathrm{F} 4 / 80+\mathrm{KCs} \\
\mathrm{IL}-1 \beta \downarrow\end{array}$ & $\begin{array}{l}\text { Liver: 8- } \\
\text { OHdG, } \\
\text { MDA } \downarrow \\
\text { Small intes- } \\
\text { tine: 8- } \\
\text { OHdG } \downarrow\end{array}$ & $\begin{array}{l}\text { Liver: lipid droplet accu- } \\
\quad \text { mulation } \downarrow\end{array}$ & & & $\begin{array}{l}\text { Hwang et al., } \\
2019^{(6)}\end{array}$ \\
\hline $\begin{array}{l}\text { Gryllus bimaculatus - } \\
\text { CPs (glycosamino- } \\
\text { glycan) }\end{array}$ & $\begin{array}{l}\text { BKS.Cg-m+/+Leprdb } \\
\text { mice, diabetes }\end{array}$ & $5 \mathrm{mg} / \mathrm{kg}$ & 1 month & $\begin{array}{l}\text { Body weight } \leftrightarrow, \\
\quad \text { abdominal fat } \leftrightarrow\end{array}$ & & $\begin{array}{l}\text { Carbonyl } \\
\text { content: } \\
\text { blood } \downarrow \text {, } \\
\text { liver } \leftrightarrow, \\
\text { GST } \leftrightarrow ;\end{array}$ & $\begin{array}{l}\text { Adipocyte density in } \\
\text { pulmons, liver and } \\
\text { kidneys } \downarrow \text { pancreas } \\
\leftrightarrow\end{array}$ & $\begin{array}{l}\text { Blood glucose } \\
\text { level } \leftrightarrow ; \\
\text { non-fasting } \\
\text { blood glu- } \\
\text { cose } \leftrightarrow(1 \\
\text { week } \downarrow)\end{array}$ & & $\begin{array}{l}\text { Ahn et al., } \\
2020^{(45)}\end{array}$ \\
\hline
\end{tabular}


Table 6. (Continued)

Animal studies

\begin{tabular}{|c|c|c|c|c|c|c|c|c|c|c|}
\hline \multirow[b]{2}{*}{ Sample } & \multirow[b]{2}{*}{ Animal/disease } & \multirow[b]{2}{*}{ Dose } & \multirow[b]{2}{*}{ Duration } & \multicolumn{6}{|c|}{ Results } & \multirow[b]{2}{*}{ References } \\
\hline & & & & $\begin{array}{l}\text { Body and organ } \\
\text { weight and compo- } \\
\text { sition }\end{array}$ & $\begin{array}{l}\text { Inflammatory } \\
\text { status }\end{array}$ & $\begin{array}{l}\text { Antioxidant/ } \\
\text { oxidant } \\
\text { markers }\end{array}$ & Lipid status & $\begin{array}{l}\text { Glycaemia/ } \\
\text { insulin status }\end{array}$ & $\begin{array}{l}\text { Coagulation } \\
\text { markers }\end{array}$ & \\
\hline & & & & & & $\begin{array}{l}\text { CAT, GPx } \\
\uparrow\end{array}$ & & $\begin{array}{l}\text { pancreatic } \\
\text { islet status, } \\
\text { liver tissue } \\
\text { status } \uparrow\end{array}$ & & \\
\hline $\begin{array}{l}\text { Gryllus bimaculatus - } \\
\text { WS }\end{array}$ & $\begin{array}{l}\text { C57BL/6Jmice, non- } \\
\text { alcoholic fatty liver } \\
\text { disease }\end{array}$ & $\begin{array}{l}100 * \\
200 * * \\
\mathrm{mg} / \mathrm{kg} / \mathrm{d}\end{array}$ & 14 weeks & $\begin{array}{l}\text { Body weight gain, } \\
\text { liver weight } \downarrow \text { adi- } \\
\text { pose tissue: } \\
\text { abdominal, kid- }\end{array}$ & 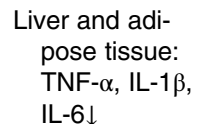 & & $\begin{array}{l}\text { TG*, TC*, LDL, HDL*, } \\
\text { NEFA } \\
\text { Liver: lipid droplet accu- } \\
\text { mulation } \downarrow\end{array}$ & & & $\begin{array}{l}\text { Im et al., } \\
\quad 2018^{(47)}\end{array}$ \\
\hline $\begin{array}{l}\text { Oxya chinensis sinu- } \\
\text { osa - WS }\end{array}$ & & & & $\begin{array}{l}\text { ney } \leftrightarrow \text {; intestinal, } \\
\text { epididymal } \downarrow\end{array}$ & $\begin{array}{l}\text { (mRNA); } \\
\text { TNF- } \alpha, \text { IL-1 } \beta \\
\downarrow \text { (expres- } \\
\text { sion) adipo- }\end{array}$ & & $\begin{array}{l}\text { TG*, TC**, LDL, } \\
\text { HDL**, NEFA } \\
\text { Liver: lipid droplet accu- } \\
\text { mulation } \downarrow\end{array}$ & $\begin{array}{l}\text { Fasting blood } \\
\text { glucose } * * \downarrow\end{array}$ & & \\
\hline $\begin{array}{c}\text { Protaetia brevitarsis } \\
\text { seulensis - WS }\end{array}$ & & & & & nectin $\uparrow \leftrightarrow \#$ & & $\begin{array}{l}\text { TG } \leftrightarrow ; \text { TC } \downarrow^{*} ; \text { LDL, } \\
\text { HDL ↔; NEFA } \downarrow \\
\text { Liver: lipid droplet accu- } \\
\text { mulation } \downarrow\end{array}$ & $\begin{array}{l}\text { Fasting blood } \\
\text { glucose } \downarrow\end{array}$ & & \\
\hline Hermetia illucens & Zebrafish, healthy & $\begin{array}{l}25,50 \\
75^{*} \\
100^{* *} \\
\%\end{array}$ & $57 d$ & $\begin{array}{l}\text { Specific growth rate } \\
\qquad \uparrow *, * * ; \text { survival } \leftrightarrow\end{array}$ & $\begin{array}{l}\text { Intestine: no } \\
\text { inflammatory } \\
\text { events (histo- } \\
\text { logical analy- } \\
\text { sis) mRNA } \\
\text { expression } \\
\text { (intestine): } \\
\text { IL-1 } 1 \beta, \text { IL-10, } \\
\text { TNF-a } \uparrow * * * *\end{array}$ & & $\begin{array}{l}\text { Liver: steatosis, fat (\%) } \\
\quad{ }^{*}, * * \text { SFA, } n-6 \uparrow ; \\
\text { MUFA, PUFA, } n-3, n- \\
9 \downarrow \text { Lipid metabolism- } \\
\text { related genes: elovl5 } \\
\uparrow^{*}, * * ; \text { elovl2, fads2 } \\
\leftrightarrow\end{array}$ & & & $\begin{array}{l}\text { Zarantoniello } \\
\text { et al, } \\
2020^{(59)}\end{array}$ \\
\hline \multirow{4}{*}{$\begin{array}{l}\text { Oxya chinensis sinu- } \\
\text { osa - } 4 \text { CPs ( } N \text {-ace- } \\
\text { tyldopamine dimers) }\end{array}$} & $\begin{array}{l}\text { C57BL/6 mice, } \\
\text { healthy }\end{array}$ & $1,2,5 \mu \mathrm{M}$ & $4 d$ & & & & & & $\begin{array}{l}\text { Blood clotting } \\
\text { time } \uparrow\end{array}$ & $\begin{array}{l}\text { Lee et al., } \\
\qquad 2017 b^{(37)}\end{array}$ \\
\hline & $\begin{array}{l}\text { C57BL/6 mice, } \\
\text { healthy }\end{array}$ & $\begin{array}{l}10,20 \\
\quad 30 \mu \mathrm{M}\end{array}$ & Acute & & & & & & & \\
\hline & $\begin{array}{l}\text { C57BL/6 mice, arterial } \\
\text { thrombosis }\end{array}$ & $\begin{array}{c}1,2^{*}, 5^{* *} \\
\mu \mathrm{M}\end{array}$ & Acute & & & & & & $\begin{array}{l}\text { Thrombi for- } \\
\text { mation } \\
\text { time } \uparrow \\
\text { thrombi } \\
\text { size } \downarrow \text { (c1; } \\
\text { c2: } *, * * ; \\
\text { c3: } *, * * ; \\
\text { c4: } * *)\end{array}$ & \\
\hline & $\begin{array}{l}\text { C57BL/6 mice, pulmo- } \\
\text { nary thrombosis }\end{array}$ & $1,2,5 \mu \mathrm{M}$ & Acute & & & & & & Mortality $\downarrow$ & \\
\hline $\begin{array}{l}\text { Oxya chinensis sinu- } \\
\text { osa-WS }\end{array}$ & $\begin{array}{l}\text { Hos/HR-1 mice, UV } \\
\text { irradiation }\end{array}$ & $\begin{array}{c}100 \mathrm{mg} / \\
\mathrm{kg} / \mathrm{d}\end{array}$ & 12 weeks & & $\begin{array}{l}\text { Skin: IL1- } \beta, \text { IL- } \\
\quad 6, \text { TNF- } \alpha \downarrow\end{array}$ & $\begin{array}{l}\text { Skin: SOD, } \\
\text { CAT } \uparrow\end{array}$ & & & & $\begin{array}{l}\text { Im et al., } \\
2019^{(55)}\end{array}$ \\
\hline $\begin{array}{l}\text { Protaetia brevitarsis - } \\
\text { WS }\end{array}$ & $\begin{array}{l}\text { C57BL/6 mice, } \\
\text { obesity }\end{array}$ & $\begin{array}{r}100,200 * \\
\mathrm{mg} / \mathrm{kg} / \mathrm{d}\end{array}$ & 7 weeks & $\begin{array}{l}\text { Body weight gain, } \\
\text { organ weight: } \\
\text { liver } \downarrow \text {, spleen } \leftrightarrow \text {; } \\
\text { fat weight: }\end{array}$ & & $\begin{array}{l}\text { Liver: GPx } \\
\quad \uparrow ; \text { CAT } \uparrow^{*}\end{array}$ & TG, TC, LDL $\downarrow$ & & & $\begin{array}{l}\text { Ahn et al., } \\
2019^{(49)}\end{array}$ \\
\hline
\end{tabular}




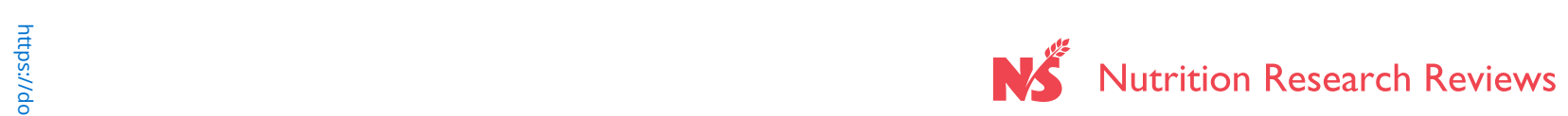

Table 6. (Continued)

\begin{tabular}{|c|c|c|c|c|c|c|c|c|c|c|}
\hline \multicolumn{11}{|l|}{ Animal studies } \\
\hline \multirow[b]{2}{*}{ Sample } & \multirow[b]{2}{*}{ Animal/disease } & \multirow[b]{2}{*}{ Dose } & \multirow[b]{2}{*}{ Duration } & \multicolumn{6}{|c|}{ Results } & \multirow[b]{2}{*}{ References } \\
\hline & & & & $\begin{array}{l}\text { Body and organ } \\
\text { weight and compo- } \\
\text { sition }\end{array}$ & $\begin{array}{l}\text { Inflammatory } \\
\text { status }\end{array}$ & $\begin{array}{l}\text { Antioxidant/ } \\
\text { oxidant } \\
\text { markers }\end{array}$ & Lipid status & $\begin{array}{l}\text { Glycaemia/ } \\
\text { insulin status }\end{array}$ & $\begin{array}{l}\text { Coagulation } \\
\text { markers }\end{array}$ & \\
\hline & & & & $\begin{array}{l}\text { epididymal, sub- } \\
\text { cutaneous } \downarrow\end{array}$ & & & & & & \\
\hline & & & & & & & $\begin{array}{l}\text { Liver: lipid droplet accu- } \\
\quad \text { mulation } \downarrow\end{array}$ & & & \\
\hline \multirow[t]{2}{*}{ Samia ricinii - LS } & $\begin{array}{l}\text { Weanling Wistar NIN } \\
\text { rats, healthy }\end{array}$ & $\begin{array}{l}10 \%(w / \\
w)\end{array}$ & 18 weeks & $\begin{array}{l}\text { Body weight gain, } \\
\text { nutrient retention, } \\
\text { organ weight } \leftrightarrow \text {. } \\
\text { Toxicity: no }\end{array}$ & & & TG, TC $\downarrow$, LDL $\uparrow$ & & & $\begin{array}{l}\text { Longvah } \\
\text { et al., } \\
2012^{(36)}\end{array}$ \\
\hline & & & & & & & Liver: TG $\leftrightarrow$ & & & \\
\hline \multirow[t]{3}{*}{$\begin{array}{l}\text { Protaetia brevitaris } \\
\text { seulensis }-5 \mathrm{CPs} \\
\text { (indole alkaloids) } \\
\text { (results are referred } \\
\text { to CPs } 1 \text { and } 2 \text { ) }\end{array}$} & $\begin{array}{l}\text { C57BL/6 mice, arterial } \\
\text { thrombosis }\end{array}$ & $\begin{array}{l}10,25 \\
50 \mu \mathrm{M}\end{array}$ & Acute & & & & & & $\begin{array}{l}\text { Arterial } \\
\text { thrombi } \\
\text { formation } \\
\text { rate and } \\
\text { size } \downarrow\end{array}$ & $\begin{array}{l}\text { Lee et al., } \\
\qquad 2017 a^{(38)}\end{array}$ \\
\hline & $\begin{array}{l}\text { C57BL/6 mice, pulmo- } \\
\text { nary thrombosis } \\
\text { model }\end{array}$ & $\begin{array}{l}10,25 \\
50 \mu \mathrm{M}\end{array}$ & Acute & & & & & & Mortality $\downarrow$ & \\
\hline & $\begin{array}{l}\text { C57BL/6 mice, } \\
\text { healthy }\end{array}$ & $\begin{array}{c}\mathrm{c} 1: 7.5, \\
14.9 \mu \mathrm{g} / \\
\text { mouse; } \\
\mathrm{c} 2: 11.5, \\
23.0 \mu \mathrm{g} / \\
\text { mouse }\end{array}$ & Acute & & & & & & $\begin{array}{l}\text { Tail bleeding } \\
\text { time } \uparrow\end{array}$ & \\
\hline \multirow[t]{2}{*}{$\begin{array}{l}\text { Tenebrio molitor-2 } \\
\text { CPs (1, cyclo(L-Pro- } \\
\text { L-Tyr) and 2, N-ace- } \\
\text { tyltyramine) }\end{array}$} & $\begin{array}{l}\text { C57BL/6 mice, arterial } \\
\text { thrombosis model }\end{array}$ & $\begin{array}{l}1,5 \\
10 \mu \mathrm{M}\end{array}$ & Acute & & & & & & $\begin{array}{l}\text { Thrombi for- } \\
\text { mation } \\
\text { time } \uparrow \\
\text { thrombi } \\
\text { size } \downarrow(\leftrightarrow) \\
\text { c2: } 1 \mu \mathrm{M})\end{array}$ & $\begin{array}{l}\text { Lee et al., } \\
\text { 2017c }\end{array}$ \\
\hline & $\begin{array}{l}\text { C57BL/6 mice, pulmo- } \\
\text { nary thrombosis } \\
\text { model }\end{array}$ & $\begin{array}{l}1,5 \\
10 \mu \mathrm{M}\end{array}$ & Acute & & & & & & $\begin{array}{l}\text { Mortality } \downarrow \text {; } \\
\text { thrombi } \\
\text { size } \downarrow(\leftrightarrow \\
\text { c2: } 1 \mu \mathrm{M})\end{array}$ & \\
\hline Tenebrio molitor & BALB/c mice, obesity & $\begin{array}{l}100 \\
3000 * \\
\mathrm{mg} / \mathrm{kg} / \mathrm{d}\end{array}$ & 6 weeks & $\begin{array}{l}\text { Body weight gain; } \\
\text { fat weight: epi- } \\
\text { didymal cell vol- } \\
\text { ume*, abdomi- } \\
\text { nal-to-peripheral } \\
\text { adipose tissue } \\
\text { volume; epididy- } \\
\text { mal white adi- } \\
\text { pose tissue } \\
\text { volume } \downarrow\end{array}$ & & & $\begin{array}{l}\text { Liver: lipid droplet accu- } \\
\quad \text { mulation } \downarrow\end{array}$ & & & $\begin{array}{l}\text { Seo et al., } \\
2017^{(48)}\end{array}$ \\
\hline
\end{tabular}


Table 6. (Continued)

\section{Human studies}

\begin{tabular}{|c|c|c|c|c|c|c|c|c|c|c|}
\hline $\begin{array}{l}\text { Edible insects- } \\
\text { extract }\end{array}$ & Animal/disease & Dose & $\begin{array}{c}\text { Treatment } \\
\text { duration }\end{array}$ & $\begin{array}{l}\text { Body and organ } \\
\text { weight and com- } \\
\text { position }\end{array}$ & $\begin{array}{l}\text { Inflammatory } \\
\text { status }\end{array}$ & $\begin{array}{c}\text { Antioxidant/ } \\
\text { oxidant } \\
\text { markers }\end{array}$ & Lipid status & $\begin{array}{c}\text { Glycaemia/ } \\
\text { insulin status }\end{array}$ & $\begin{array}{c}\text { Coagulation } \\
\text { markers }\end{array}$ & References \\
\hline Bombyx mori & Humans, healthy & $\begin{array}{l}\text { Noodles: } \\
\text { wheat } \\
\text { flour } 93 \\
\mathrm{~g}+0.83 \\
\text { g of } \\
\text { Bombyx } \\
\text { mori } \\
\text { powder }\end{array}$ & Acute & & & & & $\begin{array}{l}\text { Post-prandial } \\
\text { blood glu- } \\
\text { cose, glu- } \\
\text { cose peak, } \\
\text { IAUC glu- } \\
\text { cose, Gl } \\
\text { value } \downarrow\end{array}$ & & $\begin{array}{l}\text { Suk et al., } \\
2016^{(65)}\end{array}$ \\
\hline Cricket & Humans, healthy & $\begin{array}{l}25 \mathrm{~g} / \mathrm{d}, \text { in } \\
\text { a muffin } \\
\text { and a } \\
\text { smooth- } \\
\text { ie }\end{array}$ & $14 \mathrm{~d}$ & $\begin{array}{l}\text { Gastro-intestinal } \\
\quad \text { functionality } \leftrightarrow\end{array}$ & $\begin{array}{c}\text { slga } \leftrightarrow, \text { TNF- } \alpha \\
\text { }, \text { GM-CSF, } \\
\text { IFN } \alpha, \text { IL-1 } \alpha, \\
\text { IL-2, IL-4, IL- } \\
\text { 5, IL-6, IL-7, } \\
\text { IL-8, IL-10, } \\
\text { IL-12 (p70), } \\
\text { IL-13↔ }\end{array}$ & & $\begin{array}{l}\text { Faecal content: butyr- } \\
\text { ate } \leftrightarrow \text {, acetate, propi- } \\
\text { onate } \downarrow \text {; bile acids, } \\
\text { TG } \downarrow\end{array}$ & Glucose $\leftrightarrow$ & & $\begin{array}{r}\text { Stull et al., } \\
2018^{(66)}\end{array}$ \\
\hline
\end{tabular}

CP, compound; LPS, lipopolysaccharide; CLP, caecal ligation and puncture; TG, triacylglycerol; TC, total cholesterol; HDL, high-density lipoprotein; LS, lipo-soluble extract; TAC, total antioxidant capacity; SOD, superoxide dismutase; GPx, glutathione peroxidase; MDA, malondialdehyde, LDL, low-density lipoprotein; WS, water-soluble extract; SCFA, short-chain fatty acids; IgA, immunoglobulin A; IgG, immunoglobulin G; IgM, immunoglobulin M; NF-kB, nuclear factor-kB; IL-6, interleukin-6; TNF- $\alpha$, tumour necrosis factor- $\alpha$; 8-OHdG, 8-hydroxy-2' -deoxyguanosine; OGTT, oral glucose tolerance test; AUC, area under curve; ITT, insulin tolerance test; HbA1c, glycosylated haemoglobin concentration; HOMA-IR, homoeostasis model assessment of insulin resistance; ISI, insulin sensitivity index; TLR4, toll-like receptor 4; IL-1 $\beta$, interleukin-1 $\beta$; IFN- $\gamma$, interferon gamma; IL-4, interleukin-4; ROS, reactive oxygen species; RNS, reactive nitrogen species; GST, glutathione S-transferase; CAT, catalase; IL-10, interleukin-10; F4/80+ KCs, F4/80-positive Kupffer cells; SFA, saturated fatty acids; MUFA, monounsaturated fatty acids; PUFA, polyunsaturated fatty acids; ELOVL5, fatty acid elongase 5; ELOVL2, fatty acid elongase 2; FADS2, fatty acid desaturase 2; p-IKB- $\alpha$, phosphorylated-inhibitor of nuclear factor-kappa B-alpha; GR, glutathione reductase; NEFA, non-esterified fatty acid; ChREBP, carbohydrate-response element-binding protein; SREBP-1c, sterol regulatory element-binding protein 1C; SREBP-2, sterol regulatory element-binding protein 2; CD36, cluster of differentiation 36; FATP5, fattly acid transport protein 5; FABP1, fatty acid-binding protein 1; GPAT1, glycerol-3- phosphate acyltransferase 1; GPAT4, glycerol-3- phosphate acyltransferase 4; AGPAT1, 1-acylglycerol-3-phosphate O-acyltransferase 1; PAP1, phosphatidate phosphatase 1; DGAT1, diacylglycerol Oacyltransterase 1; ADRP, adipose differentiation-related protein; HMGCR, 3-hydroxy-3-methylglutaryl-CoA reductase; ACAT2, acetyl-CoA acetyltransferase 2; SPF, specific pathogen-free; IgE, immunoglobulin E; GI, glucose index; slgA, secretory immunoglobulin A; GM-CSF, granulocyte-macrophage colony-stimulating factor; IFN $\alpha$, interferon alpha; IL-1 $\alpha$, interleukin-1 $\alpha$; IL-2, interleukin-2. IL-5, interleukin-5; IL-7, interleukin-7; IL-8, interleukin-8; IL-12, interleukin-12; IL-13, interleukin-13. 
activator inhibitor (PAI-1) and FXa, but not tPA, in HUVECs. Moreover, in platelet-rich plasma (ex vivo) from mouse, they were able to reduce aggregation percentage and increase aPTT and $\mathrm{PT}^{(38)}$. Compounds ( $N$-acetyl dopamine dimers) extracted from Oxya chinensis sinuosa were able to increase aPTT but did not affect PT in mice plasma (ex vivo); in the same study, they also showed the ability to reduce FXa in HUVECs ${ }^{(37)}$. Lastly, two compounds (1, cyclo(L-Pro-L-Tyr) and 2, $N$-acetyltyramine) from Tenebrio molitor were able to increase aPPT and clotting time in mice blood (ex vivo) and increase endothelin1 (ET-1) production - a vasoconstrictor - in HUVECs ${ }^{(39)}$.

In four studies ${ }^{(7,47-49)}$, the ability of edible insects to modulate lipid pattern in cellular models was investigated through evaluation of lipid content or the expression of genes related to lipid metabolism. Ethanolic extract of Gryllus bimaculatus, Oxya chinensis sinuosa and Protaetia brevitarsis seulensis diminished intracellular lipid accumulation and triacylglycerol (TG) in HepG2, a human liver cancer cellular line ${ }^{(47)}$. Moreover, lipid accumulation, together with total cholesterol (TC) levels, was reduced in L-02 cells, a human fetal hepatocyte line, by polyunsaturated fatty acids (PUFAs) and $\alpha$-linolenic acid from Bombyx mori. As reported in the same paper, the extracts showed the ability to increase the mRNA and protein expression of cholesterol metabolism-related genes ${ }^{(7)}$. Incubation with Tenebrio molitor larvae decreased lipid droplet formation and TG levels in 3T3-L1 cells (murine pre-adipocytes) and mRNA expression of genes related to adipogenic differentiation and lipogenesis. Additionally, the treatment decreased phosphorylation of extracellular signal-regulated kinase (ERK) and increased phosphorylation of adenosine monophosphate-activated protein kinase- $\alpha$ (AMPK- $\alpha$ ) and p-p38 but did not affect phosphorylation of c-Jun $N$-terminal kinase (JNK) ${ }^{(48)}$. In the same cellular model, ethanolic extract of Protaetia brevitaris larvae decreased the mRNA expression of genes related to adipogenesis ${ }^{(49)}$.

Two studies investigated the effect of edible insects on inflammatory response in cellular lines. In particular, Park and colleagues $^{(46)}$ evaluated how three tetrahydroquinolines from Allomyrna dichotoma affected vascular inflammatory responses in HUVECs: they reduced vascular cell adhesion molecule-1 (VCAM-1) and intercellular adhesion molecule-1 (ICAM-1) levels, adherence of monocytes to HUVECs monolayers and migration of human neutrophils; moreover, they decreased nuclear factor- $\kappa \mathrm{B}(\mathrm{NF}-\kappa \mathrm{B})$ p 65 activity, tumour necrosis factor- $\alpha$ (TNF$\alpha)$ and interleukin-1 $\beta$ (IL-1 $\beta$ ) production and expression of phospho-p38. Furthermore, protein hydrolysates from Gryllodes sigillatus reduced NF- $\mathrm{KB}$ in RAW 264.7 cells $^{(41)}$. Aqueous extract of Locusta migratoria, tested on human peripheral blood lymphocytes, did not affect the ratio between micronucleus $(\mathrm{MN})$ and cells, sister chromatid exchange (SCE) and chromosome aberration (CA) formation ${ }^{(50)}$. Finally, in D-HMVECsm glycosaminoglycan from Gryllus bimaculatus increased vascular endothelial growth factor (VEGF), but not laminin ${ }^{(45)}$.

\section{Effect of edible insects in animal and human studies}

A total of twenty-five intervention studies in animal models and two in humans have been published, as described in Table 6. Fifteen different edible insects were investigated: five papers involved Gryllus bimaculatus ${ }^{(6,45,47,51,52)}$ and four Tenebrio molitor $^{(39,48,53,54)}$, while the use of Oxya chinensis sinuosa ${ }^{(37,47,55)}$ and Protaetia brevitaris seulensis ${ }^{(38,47,49)}$ was reported in three interventions each. One study investigated the effects of both acute and chronic settings, while four were only acute (i.e. single dose) and twenty were chronic (medium to long term) interventions. Six studies ${ }^{(36,56-60)}$ were conducted on animals that were not affected by particular stresses. Among these, Kim and colleagues investigated any toxic effects of a skimmed powder obtained from Zophobas atratus on specific pathogen-free (SPF) Sprague-Dawley rats for a 2-week repeated-dose toxicity study. Neither toxicological lesions nor mortality was observed, nor was body and organ weight influenced. The same outcomes were observed in a 13-week repeated-dose toxicity study; moreover, in macroscopic or histopathological examinations, no testsubstance-related toxicological lesions were observed ${ }^{(60)}$. No toxicity signs were observed in healthy Wistar rats after the acute ingestion of locust (Caelifera sp.) powder, which was therefore administered to the same animal model for 28 or $90 \mathrm{~d}$; organ and tissue weight, as well as TG, TC and high-density lipoprotein (HDL) cholesterol plasma levels, were not affected. However, TG and TC liver contents were increased after $90 \mathrm{~d}$ of supplementation, as faecal crude lipids. As regards caecum content, TG and TC concentration did not change, while SCFA and insulin levels were decreased ${ }^{(57)}$. The oil extracted from Samia ricinii was administered to healthy weanling Wistar National Institute of Nutrition (NIN) rats for 18 weeks, causing no variation in body weight gain or organ weight. Serum levels of TG and TC were reduced, while low-density lipoprotein (LDL) level was increased; TG content of liver was not affected ${ }^{(36)}$. Effects on lipid status were observed also in a study by Ryu ${ }^{(56)}: 5$ weeks of a diet comprising powder of whole Bombyx mori did not affect their body weight or HDL levels, but reduced TG and TC, of healthy Sprague-Dawley rats ${ }^{(56)}$. Neither body weight gain nor liver and kidney weight were altered in healthy SpragueDawley rats after 4 weeks of supplementation with grain larvae. However, their thymus and spleen weight increased, while abdominal fat was reduced. A reduced level of immunoglobulins (IgA, IgM, IgG), blood glucose and TG, TC and LDL was reported. Furthermore, this treatment modified the composition of caecal organic acids, increasing their total quantity and the acetic and propionic acid level, whilst reducing butyric, isobutyric, valeric and isovaleric acid contents ${ }^{(58)}$.

Zebrafish fed with diet containing different percentages of Hermetia illucens (75-100\% of total meal) showed a significant increase in the specific growth rate when compared with the control, while survival was not affected. Considering mRNA expression of IL-1 $\beta$, interleukin 10 (IL-10) and TNF- $\alpha$, groups fed with this percentages of insect powder showed a significant up-regulation with respect to control; furthermore, this condition induced a severe degree of steatosis. As concerns lipid metabolism-related gene expression, no significant differences in fatty acid elongase 2 (ELOVL2) or fatty acid desaturase 2 (FADS2) were detected, while fatty acid elongase 5 (ELOVL5) was upregulated. Conversely, lower doses (25-50\%) did not significantly affect the previously reported parameters. However, all the doses were able to increase the percentage of lipid categories such as saturated fatty acids (SFA) and n-6 and to decrease 
monounsaturated fatty acids (MUFA), PUFA, $n-3$ and $n-9$ in zebrafish; histological analyses of fish intestine did not show any morphological alteration or inflammatory event ${ }^{(59)}$.

Two studies ${ }^{(61,62)}$ were conducted on malnourished animals. In particular, powder of whole Acheta domesticus or Rhynchophorus phoenicis fabricius was included in the diet of malnourished weanling Sprague-Dawley rats, increasing their body weight gain, organ (spleen, right kidney, brain, liver) weight, bone mineral content and lean mass. Fat mass was increased only by Acheta domesticus; moreover, the relative biological value of the diet was improved by the insect addition ${ }^{(61)}$. Six weeks of a diet based on Gryllodes sigillatus helped malnourished mice to recover by increasing their body weight; compared with the control group, whose mice did not suffer from malnutrition, the treated mice did not show changes in levels of several inflammatory markers, such as toll-like receptor 4 (TLR4), TNF- $\alpha$, IL-1 $\beta$ and interferon $\gamma$ (IFN- $\gamma$ ), and anti-inflammatory markers, such as interleukin 4 (IL- 4 ), in spleen tissue. Similarly, leptin and adiponectin levels were not affected, while triacylglycerols were reduced $^{(62)}$.

The effect of edible insect consumption on animals fed a high-fat diet was evaluated in six studies ${ }^{(47-49,51,63,64)}$. Chitooligosaccharides from Clanis bilineata administered for 6 weeks improved the lipid status of Sprague-Dawley rats fed a high-fat diet by reducing TC, TG and LDL and increasing HDL levels. Faecal excretion of TG and TC was increased, while food efficiency ratio was decreased; although food intake remained stable, body weight gain decreased ${ }^{(63)}$

The ethanolic extracts of Protaetia brevitaris larvae, administered with a high-fat diet for 7 weeks, decreased body weight gain, epididymal and subcutaneous fat weight and liver - but not spleen - weight of obese $\mathrm{C} 57 \mathrm{BL} / 6 \mathrm{~J}$ mice; the treatment increased glutathione peroxidase (GPx) and CAT and reduced lipid droplet accumulation in mouse liver, as well as TG, TC and LDL levels ${ }^{(49)}$. Ethanolic extract of Gryllus bimaculatus, added to a high-fat diet, did not affect body weight or epididymal fat in obese rats, but it reduced total and abdominal fat weight. Moreover, it changed the composition of abdominal fat, reducing MUFA and increasing PUFA content. The supplementation did not affect TC, HDL or LDL levels, but reduced TG, IL-10 and glucose levels. Even though serum CAT was not affected, the prolonged treatment with ethanolic extract of Gryllus bimaculatus reduced protein and lipid oxidative damage caused by high-fat diet in both liver and blood, where serum uric acid and carbonyl concentrations were reduced ${ }^{(51)}$.

In a study by Im and colleagues ${ }^{(47)}$, the ethanol extracts of three different insects, that is, Gryllus bimaculatus, Oxya chinensis sinuosa and Protaetia brevitaris seulensis, were administered for 14 weeks to $\mathrm{C} 57 \mathrm{BL} / 6 \mathrm{~J}$ mice subjected to a high-fat diet to counteract the effects of non-alcoholic fatty liver disease. All the three supplementations reduced body weight gain and liver weight, together with intestinal and epididymal adipose tissue, but no significant effect was described for abdominal and kidney adipose tissue. Moreover, when inflammation markers were measured in liver and adipose tissue, mRNA levels of TNF- $\alpha$, IL-1 $\beta$ and interleukin 6 (IL-6) were found to be decreased. Oxya chinensis sinuosa and Gryllus bimaculatus reduced lipid droplet accumulation in liver, TG, TC, LDL, HDL and non- esterified fatty acid (NEFA) levels, together with fasting blood glucose, while among these markers Protaetia brevitaris seulensis reduced only lipid droplet accumulation, TC, NEFA and fasting blood glucose $\mathrm{e}^{(47)}$.

Zou et al. ${ }^{(64)}$ reported that, in Wistar rats with hypercholesterolaemia, Bombyx mori pupae oil supplementation was able to counteract the impairments induced by a high-cholesterol diet: lipid status was improved through a reduction of TC, LDL and LDL/HDL ratio without affecting TG or HDL levels. Moreover, the size of liver, kidney and heart was reduced, while that of spleen was increased. The supplementation improved antioxidant status, restoring superoxide dismutase (SOD) levels, increasing TAC levels, decreasing MDA in liver and serum and restoring the activity of GPx in rats' liver ${ }^{(64)}$. Finally, 6 weeks of treatment of obese BALB/c mice with whole powder of Tenebrio molitor resulted in a decrease in body weight gain and epididymal and abdominal-to-peripheral adipose cell volume, as well as a decrease in lipid accumulation in liver ${ }^{(48)}$.

Two studies ${ }^{(8,45)}$ investigated whether the consumption of edible insects was able to counteract the effect of diabetes in mice. The supplementation with ethanol extract of the sericin layer from the green cocoon shell of Bombyx mori improved glycaemic status in obese mice with type 2 diabetes, increasing blood glucose reduction rate and reducing blood glucose during tolerance tests, fasting blood glucose and insulin, glycosylated haemoglobin (HbA1c) and homoeostasis model assessment of insulin resistance (HOMA-IR), while improving insulin sensitivity index (ISI). Pancreas functionality was restored: islet structure and area of pancreatic beta cells and the insulin quantity secreted by them was increased. Treatment also improved antioxidant status, increasing the activity of liver GPx and SOD and reducing the liver content of MDA and 8-hydroxy-2'-deoxyguanosine (8$\mathrm{OHdG}$ ). Inflammation markers in liver, as well as infiltrations and oedema and NF-kB, IL- 6 and TNF- $\alpha$ levels, were also reduced, while body weight was not affected ${ }^{(8)}$. Glycosaminoglycan extracted from Gryllus bimaculatus and administered for 1 month to BKS.Cg-m+/+Lepr ${ }^{\mathrm{db}}$ diabetic mice, despite not affecting body weight and abdominal fat, reduced circulating levels of carbonyl. As regards antioxidant enzymes, this extract did not affect GST, but improved activity of CAT and GPx. It also reduced adipocyte density in lungs, liver and kidneys, but not in pancreas. However, the treatment improved the status of pancreas islet and liver tissue that was damaged by the diabetic condition. Blood glucose levels were not affected, whilst non-fasting blood glucose was reduced only after 1 week of treatment ${ }^{(45)}$.

The effect of edible insects against stress induced by alcohol consumption was evaluated in three different studies ${ }^{(6,53,54)}$. The first reported positive action of the aqueous extract of Gryllus bimaculatus in restoring the normal physiological levels of 8OHdG levels and MDA content in liver and small intestine of C57BL/6J mice with liver damage caused by acute alcohol exposure; liver droplet accumulation was also reduced, as well as levels of inflammation markers (F4/80-positive Kupffer cells (F4/ $80+\mathrm{KCs})$ and $\mathrm{IL}-1 \beta)^{(6)}$. The treatment with fermented Tenebrio molitor powder of Sprague-Dawley rats, impaired by a chronic alcohol diet, reduced liver weight and increased activity of glutathione reductase (GR) and total and reduced GPx hepatic content, but no effect was observed on SOD and CAT 
levels. Moreover, phosphorylated-inhibitor of nuclear factorkappa B-alpha (p-IкB- $\alpha$ ) levels were reduced, as well as serum and liver content of TNF- $\alpha$, but not of IL- 6 . As regards lipid status, hepatic levels of TG, NEFA and TC were also reduced, as alcohol-induced hepatic lipid accumulation, focal necrosis and mild fibrosis were attenuated. Also, expression of lipid synthesis transcription factors, triacylglycerol synthesis-related genes and cholesterol synthesis and esterification-related genes was reduced, while expression of fatty acid uptake and transport-related genes was not affected. ${ }^{(53)}$. The treatment with fermented defatted Tenebrio molitor powder of Sprague-Dawley rats fed with a chronic alcohol diet dose-dependently increased hepatic $\beta$ oxidation $^{(54)}$

Concerning the effect on coagulation markers ${ }^{(37-39)}$, compounds extracted from Protaetia brevitaris seulensis ${ }^{(38)}$, Oxya chinensis sinuosa $^{(37)}$ and Tenebrio molitor ${ }^{(39)}$ decreased arterial thrombi formation rate and size in mice with arterial thrombosis and decreased mortality in mice with pulmonary thrombosis; in this model, 1, cyclo(L-Pro-L-Tyr) and 2, $\mathrm{N}$-acetyltyramine from Tenebrio molitor were also able to reduce thrombi size ${ }^{(39)}$. Indole alkaloids and $N$-acetyldopamine dimers extracted respectively from Protaetia brevitaris seulensis ${ }^{(38)}$ and Oxya chinensis sinuosa ${ }^{(37)}$ increased tail bleeding time in healthy mice.

Edible insects were also able to improve antioxidant and inflammatory status that was previously impaired by different stressing agents such as sepsis ${ }^{(46)}$, varicocele ${ }^{(52)}$ or UV radiation $^{(55)}$. Four tetrahydroquinolines from Allomyrina dichotoma were administered twice to caecal ligation and puncture (CLP)-induced septic C57BL/6 mice, resulting in an increase in the survival rate. There were no significant differences between the lungs of the treated and untreated mice: interstitial oedema was observed, and the pulmonary architecture was severely damaged. Moreover, to evaluate effect of compounds against inflammation, lipopolysaccharide (LPS)-induced vascular permeability in mice was inhibited by the tetrahydroquinolines ${ }^{(1-}$ 3), which reduced LPS-induced leucocyte migration into the murine peritoneal cavities ${ }^{(46)}$. Moreover, treatment with Gryllus bimaculatus improved antioxidant and anti-inflammatory status in testicular tissue of Sprague-Dawley rats affected by varicocele. Furthermore, the increased levels of MDA, ROS and reactive nitrogen species (RNS) were significantly reduced $^{(52)}$. Finally, the extract of Oxya chinensis sinuosa, administered to Hos/HR-1 hairless mice for 12 weeks, reduced the damage induced by UV irradiation as well as inflammation markers (IL-1- $\beta$, IL- 6 and TNF- $\alpha$ ), while the activity of SOD and CAT was increased ${ }^{(55)}$.

Evidence in humans is available only for two dietary intervention trials. Wheat noodles enriched with Bombyx mori powder, were provided to thirteen healthy humans, fasting in the morning, following a cross-over acute ingestion design. The Bombyx mori noodles significantly reduced post-prandial blood glucose, glucose peak, area under the curve (AUC) of glucose and glucose index (GI) compared with control noodles ${ }^{(65)}$. In a recent chronic intervention study, $25 \mathrm{~g} / \mathrm{d}$ of dried roasted cricket powder, included in a muffin and in a dry mix shake, was given to twenty healthy humans for breakfast for $14 \mathrm{~d}$ following a cross-over design. The treatment did not modify intestinal microbiota, gastrointestinal functionality, glycaemia or IgA levels of the subjects. However, TNF- $\alpha$ plasma levels decreased, as did the acetate, propionate, bile acids and TG content of faeces ${ }^{(66)}$.

\section{Discussion}

In this work, we summarised the body of evidence on selected functional properties of edible insects in modulating oxidative and inflammatory stress, platelet aggregation, lipid and glucose metabolism and weight control in different experimental models. Concerning the considered species, among a total of fortythree edible insects, the Gryllidae family and Tenebrio molitor were the most investigated, respectively in seventeen and sixteen studies. Both species are included in the list of insects for human consumption from the European Food Safety Authority and are also widely present in the market. Regarding the different aspects, antioxidant properties were investigated in thirty-six different species (Tenebrio molitor and Gryllidae in fourteen and twelve studies, respectively); eleven species were tested for their ability to affect lipid status (Gryllus bimaculatus and Bombyx mori in four studies and Tenebrio molitor in three studies). As regards the anti-inflammatory properties, the effects of nine insects were investigated: Gryllus bimaculatus was cited in five research articles, whilst Grillodes sigillatus and Bombyx mori were tested in two studies each. Gryllus bimaculatus was investigated for its ability to modulate glucose metabolism in four different studies, followed by Gryllodes sigillatus with two studies. Lastly, the studies involving coagulation markers investigated seven insects, of which Tenebrio molitor and Protaetia brevitaris seulensis were evaluated twice each. It is noticeable that the Gryllidae family, represented mainly by Gryllus bimaculatus and Gryllodes sigillatus, is the most cited in all the topics, the only exception being the effect on coagulation. Moreover, one of the two studies on human subjects involved crickets of the Gryllidae family, while the other study was focused on Bombyx mori.

The antioxidant properties of edible insects were investigated in thirty-six out of fifty-five studies involving animals ( $n$ $=11)$, cell cultures $(n=9)$ or in vitro $(n=22)$. Results clearly show that all insects tested in vitro and in cellular models displayed radical scavenging or metal ion chelation properties, as well as the ability to modulate glutathione $S$-transferase and catalase, with activity depending on the utilised concentration. Also, the findings in animal models were consistent with in vitro results, supporting the antioxidant properties of edible insects. According to the different studies, the effect was evident in serum, liver and skin, with a mechanism of action ranging from the increase in antioxidant capacity up to the modulation of endogenous antioxidant enzymes. In the majority of the studies, the antioxidant effect was more evident when specific stressors such as high-fat diet, oxidative stress, obesity, alcoholic liver disease or UV irradiation were present. Although the antioxidant properties have been widely investigated in vitro and in cellular and animal models, studies on human subjects are lacking. Overall, the evidence from the available studies clearly showed that all the tested insects, with varying ability, were able to reduce an induced oxidative stress, modulating redox status of cellular and body fluids and restoring the impaired activity of antioxidant enzymes. 
The effect on lipid markers was evaluated in twenty-one research articles; the studies were carried out mostly in animal models $(n=18)$, while only few were in cellular models $(n=4)$ or in vitro $(n=1)$. When markers of dyslipidaemia were evaluated in animal studies, edible insects were able to reduce TG (10/ 12), TC (7/11) and LDL (6/8) levels, without affecting HDL (5/7), in body fluids and tissues. Moreover, the reduction of TG was detected also in hepatocytes and adipocytes, while a study reported a reduction of TC in hepatocytes. Reduction of lipid droplets in liver was detected in animal models (7/7) and in two studies on cellular model of hepatocytes, suggesting an effect on liver steatosis. A reduction in fat tissue weight or volume was detected in five out of seven studies, together with a change in fat composition (2/2). Furthermore, edible insects positively modulated lipid metabolism and fat accumulation in animal $(n=2)$ and cellular models $(n=3)$. The only in vitro study evaluating the ability of edible insects to affect lipid metabolism reported an inhibition of pancreatic lipase, with the possible consequence of preventing the breakdown of triacylglycerol and delaying the absorption of fatty acids ${ }^{(67)}$. Furthermore, the effect of consumption of edible insects on weight control, expressed as body weight (BW) or body weight gain (BWG) was evaluated in fifteen studies; considering both parameters, either an increase $(2 / 15)$ or a decrease $(4 / 15)$ was reported, but in most of the cases no significative effect was observed (9/15). However, if the results are classified on the basis of the type of diet or the body weight at the baseline, the supplementation with edible insects induced a decrease in BW or BWG in four out of five cases in obese animals or following a high-fat diet, while an increase of BW was reported when malnourished animals were considered $(n=2)$. Based on the evidence from animal studies, dietary intervention with edible insects reduced TC, TG and LDL, while the effect on BW was dependent on whether animals were obese or malnourished.

The anti-inflammatory properties of edible insects were evaluated in sixteen papers, twelve in vivo and five in cellular models. Concentrations of cytokines were evaluated in nine in vivo studies, leading to a reduction in circulating levels increased by different stressors (8/9). An increase in cytokine levels was observed only when high doses of Hermetia illucens ( $75-100 \%$ of the total diet) were given to healthy zebrafish $^{(59)}$, although no inflammatory events were observed through histological analysis. An effect on reducing circulating levels of TNF- $\alpha$ was shown in humans, although we need to consider that the study was conducted on healthy subjects, who may have low or scarce levels of inflammation, and that the number of subjects enrolled was low ${ }^{(66)}$. Effects on immunoglobulins were evaluated in two papers: IgE levels - as an identifier of allergic reactions in rats - were not detected, whilst an increase in IgG, IgA and IgM was revealed, considered by the authors to be a bifidogenic effect from the antimicrobial peptides in the used extract. NF- $\kappa \mathrm{B}$ levels, transcription-factor-regulating genes involved in inflammatory responses, were decreased in cellular models, HUVECs and RAW 264.7, and in an animal study. However, TLR4 levels, whose stimulation leads to NF- $\mathrm{BB}$ activation, were not affected. Concerning the effect on specific organs, reduction of inflammatory infiltrations and oedema in liver, leucocyte migration and vascular permeability in peritoneum were observed. Finally, three studies showed activity in reducing NO production in macrophages, a radical involved in the modulation of inflammation and immunity ${ }^{(68)}$. Therefore, the evidence from cellular and animal models supports an effect on reducing inflammatory cytokines thorough the modulation of NF- $\mathrm{BB}$ levels, without affecting immunoglobulins.

Effects of edible insects on glucose and/or insulin status were evaluated in nine studies; six out of the total were in vivo interventions, and three were in vitro studies. Most of the in vivo studies reported improvements in diabetes markers, such as reduced blood glucose (4/6) and serum insulin (2/2), as well as an enhancement of pancreas structure and functionality $(2 / 2)$. Moreover, a reduction in glycated haemoglobin (Hba1c), a tool in both routine management and diagnosis of diabetes ${ }^{(69)}$, was detected. However, only two interventions out of six involved diabetic mice. All the in vitro studies outlined a positive effect through the inhibition of DPP-IV and $\alpha$-glucosidase, suggesting a role in modulating glucose homoeostasis ${ }^{(70)}$. The results in vitro and in animal models are supported by evidence in humans showing an effect of noodles enriched with Bombyx mori powder on reducing glycaemic index ${ }^{(65)}$. Although further evidence is needed, results suggest that edible insects can modulate glycaemia/insulin homoeostasis with potential effect on the management of glycaemic index, a key aspect for diabetes prevention.

Anti-coagulation properties of edible insects have been investigated in only four different works: three out of four studies were performed by the same research group, investigating compounds extracted from one insect for each, using either in vitro, ex vivo, cellular or animal models vivo with acute interventions, while the other study investigated in vitro the properties of seven different insects. The selected edible insects showed anti-coagulant properties in vitro and ex vivo, by reducing aPTT and PT both measuring speed of blood clotting - and affecting the coagulation cascade by modulation of factor $\mathrm{X}$ and thrombin. Moreover, a reduction in PAI-1 was recorded, whose inhibition may reduce the incidence of thrombotic events ${ }^{(71)}$. PAI- 1 is also an important physiological inhibitor of $\mathrm{tPA}^{(72)}$ : this molecule, whose main function within the vascular system is the removal of fibrin ${ }^{(73)}$, was not affected by in vitro treatment with compounds extracted from edible insects. When compounds derived from edible insects were administered to mice in acute interventions, a decrease of thrombi formation and consequent mortality in mice models of arterial or pulmonary thrombosis was observed; moreover, these compounds prolonged tail bleeding time in healthy mice. Overall, the available literature is scarce and from one research group only, making it hard to draw any conclusion on the anti-platelet aggregation properties of edible insects.

In terms of research quality of the results, the evidence from in vitro, cellular and animal studies is solid because the markers and biomarkers utilised are appropriate and the animal studies are properly designed. Human studies involved a low number of subjects, short period of dietary intervention and lack of a 
dietary assessment of the subjects during the studies, making it difficult to draw any conclusions about the clinical relevance of the findings.

A few more considerations are needed: first of all, different species display similar activities such as antioxidants and antiinflammatory properties, despite being characterised by different genes, habitat, physiological needs and eating habits, making it difficult to associate the specific functional role to the different species. Studies assessing the functional properties of different species characterised by dietary habits and environment, as well as the effect of different feedings, will help us to understand the variables influencing the functional properties of the insects.

Secondly, the majority of the studies tested extracts without identifying the compounds responsible for the functional effect or without testing sub-fractions with different chemical composition. However, although functional properties of the edible insects might be related to their variegate molecular composition, as occurs for foods, the identification of the single molecules is a necessary step for nutraceutical or pharmacological purposes.

Thirdly, the evidence from in vitro, cellular and animal models on the functional properties reviewed in this manuscript is based on robust and reliable markers with a statistical significance. However, the scarce evidence in humans highlights the urgent need for long-term dietary intervention trials to endorse the role of edible insects as functional foods. The step of human consumption is critical because there is no information on the bioavailability of functional molecules from edible insects in body fluids following consumption. Moreover, it would be interesting to understand the effect of intestinal microbiota on molecules from the digestion processes of edible insects and if the arising metabolites are endowed with functional properties. In this view, it would be interesting to develop observational or epidemiological studies assessing biomarkers related to antioxidant, inflammatory and immune status as well as microbiota analyses in populations where insects are a part of the usual diet.

The inclusion of edible insects in the category of functional food might open a new scenario for food industries through the enrichment of food with proteins, extracts or flour obtained from edible insects, representing a novel aspect with scientific, commercial and social impacts on society in the following years. However, for such an approach to be efficacious, it is important to investigate consumers' acceptance, mainly in Western countries, of insect-based foods ${ }^{(74)}$. This is a process that requires time and campaigning of information for consumers, focusing on the importance of reducing the ecological impact of animal-based products with more sustainable and functional foods.

\section{Conclusions}

In conclusion, based on the available body of evidence, mainly in vitro, cellular and animal studies, edible insects are a promising source of bioactive ingredients endowed with antioxidant and anti-inflammatory properties, involved in the modulation of glucose and lipid metabolism, potentially leading to health benefits. However, dietary intervention trials are urgently needed to confirm and define the efficacy of edible insects as functional foods in humans, confirming the promising evidence from in vitro and animal models. We think that this review could promote research on the functional properties of edible insects, amplifying our knowledge on this topic and ensuring that correct information is relayed from the media to consumers.

\section{Author contributions}

M.S. conceived the topic of the review and supervised the work. V.D.A. wrote the initial draft. N.B., G.S. and C.D.M. revised the work. All authors approved the submitted version.

\section{Financial support}

This research received no specific grant from any funding agency, commercial or not-for-profit sectors.

\section{Conflict of interest}

None.

\section{References}

1. FAO. (2017) The Future of Food and Agriculture - Trends and Challenges. Rome. Available at: http://www.fao.org/3/i6583e/ i6583e.pdf

2. de Carvalho NM, Madureira AR \& Pintado ME (2020). The potential of insects as food sources - a review. Crit Rev Food Sci Nutr. Published online: 29 November 2020. doi: 10.1080/ 10408398.2019.1703170.

3. Ercolini D \& Fogliano V (2018) Food design to feed the human gut microbiota. J Agric Food Chem. Published online: 5 April 2018. doi: 10.1021/acs.jafc.8b00456.

4. Costa-Neto EM (2005) Entomotherapy, or the medicinal use of insects. J Ethnobiol 25, 93-114.

5. D'Antonio V, Serafini M \& Battista N (2021) Dietary modulation of oxidative stress from edible insects: a mini-review. Front Nutr. Published online: 26 February 2021. doi: 10.3389/fnut. 2021.642551.

6. Hwang BB, Chang MH, Lee JH, et al. (2019) The edible insect Gryllus bimaculatus protects against gut-derived inflammatory responses and liver damage in mice after acute alcohol exposure. Nutrients. Published online 16 April 2019. doi: 10.3390/ nu11040857.

7. Luo Y, Wang L, Lv Y, et al. (2020) Regulation mechanism of silkworm pupa oil PUFAs on cholesterol metabolism in hepatic cell L-02. J Sci Food Agric 100, 1418-1425.

8. Zhao J-G, Wang H-Y, Wei Z-G, et al. (2019) Therapeutic effects of ethanolic extract from the green cocoon shell of silkworm Bombyx mori on type 2 diabetic mice and its hypoglycaemic mechanism. Toxicol Res. Published online: 26 February 2019. doi: $10.1039 / \mathrm{c} 8 \mathrm{tx} 00294 \mathrm{k}$.

9. Nowakowski AC, Miller AC, Miller ME, et al. (2021) Potential health benefits of edible insects. Crit Rev Food Sci Nutr. Published online: 5 January 2021. doi: 10.1080/10408398. 2020.1867053.

10. Patel S, Suleria HAR \& Rauf A (2019) Edible insects as innovative foods: nutritional and functional assessments. Trends Food Sci Technol 86, 352-359.

11. Jantzen da Silva Lucas A, Menegon de Oliveira L, da Rocha M, et al. (2020) Edible insects: an alternative of nutritional, 
functional and bioactive compounds. Food Chem. Published online: 13 December 2019. doi: 10.1016/j.foodchem.2019. 126022.

12. Ojha S, Bekhit AE-D, Grune T, et al. (2021) Bioavailability of nutrients from edible insects. Curr Opin Food Sci 41, 240-248.

13. Stull VJ (2021) Impacts of insect consumption on human health. J Insects Food Feed 7, 695-713.

14. Messina CM, Gaglio R, Morghese M, et al. (2019) Microbiological profile and bioactive properties of insect powders used in food and feed formulations. Foods. Published online: 9 September 2019. doi: 10.3390/foods8090400.

15. Zielińska E, Baraniak B \& Kara囚 M (2017) Antioxidant and antiinflammatory activities of hydrolysates and peptide fractions obtained by enzymatic hydrolysis of selected heat-treated edible insects. Nutrients $\mathbf{9}, 970$.

16. Mintah BK, He R, Dabbour M, et al. (2019) Techno-functional attribute and antioxidative capacity of edible insect protein preparations and hydrolysates thereof: effect of multiple mode sonochemical action. Ultrason Sonochem. Published online: 05 July 2019. doi: 10.1016/j.ultsonch.2019.104676.

17. Mintah BK, He R, Dabbour M, et al. (2019) Sonochemical action and reaction of edible insect protein: influence on enzymolysis reaction-kinetics, free-Gibbs, structure, and antioxidant capacity. J Food Biochem. Published online: 18 September 2019. doi: 10.1111/jfbc. 12982.

18. Haber M, Mishyna M, Itzhak Martinez JJ, et al. (2019) Edible larvae and pupae of honey bee (Apis mellifera): odor and nutritional characterization as a function of diet. Food Chem $\mathbf{2 9 2}$, 197-203.

19. Alves AV, Argandoña EJS, Linzmeier AM, et al. (2016) Chemical composition and food potential of Pachymerus nucleorum larvae parasitizing Acrocomia aculeata kernels. PLoS One. Published online: 31 March 2016. doi: 10.1371/journal.pone. 0152125

20. Son YJ, Choi SY, Hwang IK, et al. (2020) Could defatted mealworm (Tenebrio molitor) and mealworm oil be used as food ingredients? Foods $\mathbf{9}, 40$

21. Mancini S, Fratini F, Turchi B, et al. (2019) Former foodstuff products in Tenebrio molitor rearing: effects on growth, chemical composition, microbiological load, and antioxidant status. Animals. Published online: 25 July 2019. doi: 10.3390/ ani9080484.

22. Tang Y, Debnath T, Choi EJ, et al. (2018) Changes in the amino acid profiles and free radical scavenging activities of Tenebrio molitor larvae following enzymatic hydrolysis. PLoS One 13, e0196218.

23. Flores DR, Casados LE, Velasco SF, et al. (2020) Comparative study of composition, antioxidant and antimicrobial activity of two adult edible insects from Tenebrionidae family. $B M C$ Chem. Published online: 9 September 2020. doi: 10.1186/ s13065-020-00707-0.

24. Dutta P, Dey T, Manna P, et al. (2016) Antioxidant potential of Vespa affinis L., a traditional edible insect species of North East India. PLoS One. Published online: 19 May 2016. doi: 10.1371/ journal.pone.0156107.

25. Botella-Martínez C, Lucas-González R, Pérez-Álvarez JA, et al. (2020) Assessment of chemical composition and antioxidant properties of defatted flours obtained from several edible insects. Food Sci Technol Int. Published online: 22 September 2020. doi: 10.1177/1082013220958854.

26. Di Mattia C, Battista N, Sacchetti G, et al. (2019) Antioxidant activities in vitro of water and liposoluble extracts obtained by different species of edible insects and invertebrates. Front Nutr 6, 106.

27. Shantibala T, Lokeshwari RK \& Debaraj H (2014) Nutritional and antinutritional composition of the five species of aquatic edible insects consumed in Manipur, India. I Insect Sci. Published online: 26 January 2014. doi: 10.1093/jis/14.1.14.

28. Pyo SJ, Kang DG, Jung C, et al. (2020) Anti-thrombotic, anti-oxidant and haemolysis activities of six edible insect species. Foods 9, 401 .

29. Sousa P, Borges S \& Pintado M (2020) Enzymatic hydrolysis of insect: Alphitobius diaperinus towards the development of bioactive peptide hydrolysates. Food Funct 11, 3539-3548.

30. Navarro del Hierro J, Gutiérrez-Docio A, Otero P, et al. (2020) Characterization, antioxidant activity, and inhibitory effect on pancreatic lipase of extracts from the edible insects Acheta domesticus and Tenebrio molitor. Food Chem 309, e125742.

31. Anuduang A, Loo YY, Jomduang S, et al. (2020) Effect of thermal processing on physico-chemical and antioxidant properties in mulberry silkworm (Bombyx mori L.) powder. Foods. Published online: 3 July 2020. doi: 10.3390/foods9070871.

32. Dutta P, Dey T, Dihingia A, et al. (2017) Antioxidant and glucose metabolizing potential of edible insect, Brachytrupes orientalis via modulating Nrf2/AMPK/GLUT4 signaling pathway. Biomed Pharmacother 95, 556-563.

33. Sun M, Xu X, Zhang Q, et al. (2018) Ultrasonic-assisted aqueous extraction and physicochemical characterization of oil from Clanis bilineata. J Oleo Sci 67, 151-165.

34. Hall F, Johnson PE \& Liceaga A (2018) Effect of enzymatic hydrolysis on bioactive properties and allergenicity of cricket (Gryllodes sigillatus) protein. Food Chem 262, 39-47.

35. Anuduang A, Loo YY, Jomduang S, et al. (2020) Effect of thermal processing on physico-chemical and antioxidant properties in mulberry silkworm (Bombyx mori L.) powder. Foods 9, 871 .

36. Longvah T, Manghtya K \& Qadri SSYH (2012) Eri silkworm: a source of edible oil with a high content of $\alpha$-linolenic acid and of significant nutritional value. J Sci Food Agric 92, 1988-1993.

37. Lee W, Lee HS, Kim MA, et al. (2017) Evaluation of novel factor $\mathrm{Xa}$ inhibitors from Oxya chinensis sinuosa with anti-platelet aggregation activity. Sci Rep. Published online: 11 August 2017. doi: 10.1038/s41598-017-08330-1.

38. Lee JI, Lee W, Kim MA, et al. (2017) Inhibition of platelet aggregation and thrombosis by indole alkaloids isolated from the edible insect Protaetia brevitarsis seulensis (Kolbe). J Cell Mol Med 21, 1217-1227.

39. Lee W, Kim MA, Park IW, et al. (2017) Novel direct factor Xa inhibitory compounds from Tenebrio molitor with anti-platelet aggregation activity. Food Chem Toxicol 109, 19-27.

40. Yoon S, Wong NAK, Chae M, et al. (2019) Comparative characterization of protein hydrolysates from three edible insects: mealworm larvae, adult crickets, and silkworm pupae. Foods 8, 563.

41. Hall F, Reddivari L \& Liceaga AM (2020) Identification and characterization of edible cricket peptides on hypertensive and glycemic in vitro inhibition and their anti-inflammatory activity on RAW 264.7 macrophage cells. Nutrients 12, 3588.

42. De Carvalho NM, Teixeira F, Silva S, et al. (2019) Potential prebiotic activity of Tenebrio molitor insect flour using an optimized in vitro gut microbiota model. Food Funct 10, 3909-3922.

43. Koc K, Incekara U \& Turkez H (2014) Biomonitoring of the genotoxic effects and oxidative potentials of commercial edible dung beetles (Onitis sp.), grasshopper (Caelifera sp.) and mole crickets (Gryllotalpa sp.) in vitro. Toxicol Ind Health 30, 683-689.

44. Memi冈 E, Türkez H, \ncekara Ü, et al. (2013) In vitro biomonitoring of the genotoxic and oxidative potentials of two commonly eaten insects in southwestern Nigeria. Toxicol Ind Health 29, 52-59.

45. Ahn MY, Kim BJ, Kim HJ, et al. (2020) Anti-diabetic activity of field cricket glycosaminoglycan by ameliorating oxidative stress. BMC Complement Med Ther 20, 232. 
46. Park I, Lee W, Yoo Y, et al. (2020) Protective effect of tetrahydroquinolines from the edible insect Allomyrina dichotoma on LPS-induced vascular inflammatory responses. Int $\mathrm{J} \mathrm{Mol}$ Sci 21, 3406.

47. Im AR, Yang WK, Park YC, et al. (2018) Hepatoprotective effects of insect extracts in an animal model of nonalcoholic fatty liver disease. Nutrients $\mathbf{1 0}, 735$.

48. Seo M, Goo TW, Chung MY, et al. (2017) Tenebrio molitor larvae inhibit adipogenesis through AMPK and MAPKs signaling in 3T3-L1 adipocytes and obesity in high-fat diet-induced obese mice. Int J Mol Sci 18, 518.

49. Ahn EM, Myung NY, Jung HA, et al. (2019) The ameliorative effect of Protaetia brevitarsis larvae in HFD-induced obese mice. Food Sci Biotechnol. Published online: 4 January 2019. doi: 10.1007/s10068-018-00553-w.

50. Turkez H, Øncekara Ü, Güner A, et al. (2014) The cytogenetic effects of the aqueous extracts of migratory locust (Locusta migratoria L.) in vitro. Toxicol Ind Health 30, 233-237.

51. Ahn MY, Kim MJ, Kwon RH, et al. (2015) Gene expression profiling and inhibition of adipose tissue accumulation of $G$. bimaculatus extract in rats on high fat diet. Lipids Health Dis. Published online: 24 September 2015. doi: 10.1186/s12944015-0113-3.

52. Karna KK, Choi NY, Kim CY, et al. (2020) Gui-A-Gra attenuates testicular dysfunction in varicocele-induced rats via oxidative stress, ER stress and mitochondrial apoptosis pathway. Int $J$ Mol Sci 21, 9231.

53. Choi RY, Ham JR, Ryu HS, et al. (2020) Defatted Tenebrio molitor larva fermentation extract modifies steatosis, inflammation and intestinal microflora in chronic alcohol-fed rats. Nutrients 12, 1426.

54. Choi RY, Ji M, Lee MK, et al. (2020) Metabolomics study of serum from a chronic alcohol-fed rat model following administration of defatted Tenebrio molitor larva fermentation extract. Metabolites 10, 436.

55. Im AR, Park I, Ji KY, et al. (2019) Protective effects of Oxya chinensis sinuosa Mishchenko against ultraviolet B-induced photodamage in hairless mice. BMC Complement Altern Med 19, 286.

56. Ryu SP (2014) Silkworm pupae powder ingestion increases fat metabolism in swim-trained rats. J Exerc Nutr Biochem 18, 141149.

57. Ochiai M, Inada M \& Horiguchi S (2020) Nutritional and safety evaluation of locust (Caelifera) powder as a novel food material. J Food Sci 85, 279-288.

58. Park SO \& Park BS (2015) Bifidogenic effect of grain larvae extract on serum lipid, glucose and intestinal microflora in rats. J Biosci 40, 513-520.

59. Zarantoniello M, Randazzo B, Gioacchini G, et al. (2020) Zebrafish (Danio rerio) physiological and behavioural responses to insect-based diets: a multidisciplinary approach. Sci Rep. Published online: 30 June 2020. doi: 10.1038/s41598020-67740-w.
60. Kim SY, Kwak KW, Park ES, et al. (2020) Evaluation of subchronic oral dose toxicity of freeze-dried skimmed powder of Zophobas atratus larvae (frpfdZAL) in rats. Foods $\mathbf{9}, 995$.

61. Agbemafle I, Hanson N, Bries AE, et al. (2019) Alternative protein and iron sources from edible insects but not Solanum torvum improved body composition and iron status in malnourished rats. Nutrients 11, 2481.

62. Bergmans RS, Nikodemova M, Stull VJ, et al. (2020) Comparison of cricket diet with peanut-based and milk-based diets in the recovery from protein malnutrition in mice and the impact on growth, metabolism and immune function. PLoS One. Published online: 11 June 2020. doi: 10.1371/journal. pone.0234559.

63. Xia Z, Chen J \& Wu S. (2013) Hypolipidemic activity of the chitooligosaccharides from Clanis bilineata (Lepidoptera), an edible insect. Int J Biol Macromol 59, 96-98.

64. Zou Y, Hu T, Shi Y, et al. (2016) Silkworm pupae oil exerts hypercholesterolemic and antioxidant effects in high-cholesterol diet-fed rats. I Sci Food Agric. Published online: 26 September 2016. doi: 10.1002/jsfa.8009.

65. Suk W, Kim JE, Kim DY, et al. (2016) Effect of wheat flour noodles with Bombyx mori powder on glycemic response in healthy subjects. Prev Nutr Food Sci 21, 165-170.

66. Stull VJ, Finer E, Bergmans RS, et al. (2018) Impact of edible cricket consumption on gut microbiota in healthy adults, a double-blind, randomized crossover trial. Sci Rep. Published online 17 July 2018. doi: 10.1038/s41598-018-29032-2.

67. Rajan L, Palaniswamy D \& Mohankumar SK (2020) Targeting obesity with plant-derived pancreatic lipase inhibitors: a comprehensive review. Pharmacol Res. Published online: 8 February 2020. doi: 10.1016/j.phrs.2020.104681.

68. Lee M, Rey K, Besler K, et al. (2017) Immunobiology of nitric oxide and regulation of inducible nitric oxide synthase. Results Probl Cell Differ 62, 181-207.

69. Weykamp C (2013) HbA1c: a review of analytical and clinical aspects. Ann Lab Med. Published online: 17 October 2013. doi: 10.3343/alm.2013.33.6.393.

70. De S, Banerjee S, Kumar SKA, et al. (2019) Critical role of dipeptidyl peptidase IV: a therapeutic target for diabetes and cancer. Mini Rev Med Chem 19, 88-97.

71. Gils A \& Declerck PJ (2004) Plasminogen activator inhibitor-1. Curr Med Chem 11, 2323-2334.

72. Pant A, Kopec AK, Baker KS, et al. (2018) Plasminogen activator inhibitor-1 reduces tissue-type plasminogen activator-dependent fibrinolysis and intrahepatic hemorrhage in experimental acetaminophen overdose. Am J Pathol. Published online: 16 February 2018. doi: 10.1016/j.ajpath.2018.01.010.

73. Kruithof EKO \& Dunoyer-Geindre S (2014) Human tissue-type plasminogen activator. Thromb Haemost. Published online: 10 April 2014. doi: 10.1160/TH13-06-0517.

74. Skotnicka M, Karwowska K, Kłobukowski F, et al. (2021) Possibilities of the development of edible insect-based foods in Europe. Foods. Published online: 3 Apr 2021. doi: 10.3390/ foods10040766. 